\title{
The flaring HI disk of the nearby spiral galaxy NGC $2683^{\star}, \star \star$
}

\author{
B. Vollmer, F. Nehlig, and R. Ibata
}

\author{
Observatoire astronomique de Strasbourg, UMR 7750, 11, rue de 1'Université, 67000 Strasbourg, France \\ e-mail: Bernd.Vollmer@astro.unistra.fr
}

Received 23 October 2013 / Accepted 12 November 2015

\begin{abstract}
New deep VLA D array HI observations of the highly inclined nearby spiral galaxy NGC 2683 are presented. Archival C array data were processed and added to the new observations. To investigate the $3 \mathrm{D}$ structure of the atomic gas disk, we made different 3D models for which we produced model HI data cubes. The main ingredients of our best-fit model are (i) a thin disk inclined by $80^{\circ}$; (ii) a crude approximation of a spiral and/or bar structure by an elliptical surface density distribution of the gas disk; (iii) a slight warp in inclination between $10 \mathrm{kpc} \leq R \leq 20 \mathrm{kpc}$ (decreasing by $10^{\circ}$ ); (iv) an exponential flare that rises from $0.5 \mathrm{kpc}$ at $R=9 \mathrm{kpc}$ to $4 \mathrm{kpc}$ at $R=15 \mathrm{kpc}$, stays constant until $R=22 \mathrm{kpc}$, and decreases its height for $R>22 \mathrm{kpc}$; and (v) a low surface-density gas ring with a vertical offset of $1.3 \mathrm{kpc}$. The slope of NGC 2683's flare is comparable, but somewhat steeper than those of other spiral galaxies. NGC 2683's maximum height of the flare is also comparable to those of other galaxies. On the other hand, a saturation of the flare is only observed in NGC 2683. Based on the comparison between the high resolution model and observations, we exclude the existence of an extended atomic gas halo around the optical and thin gas disk. Under the assumption of vertical hydrostatic equilibrium we derive the vertical velocity dispersion of the gas. The high turbulent velocity dispersion in the flare can be explained by energy injection by (i) supernovae; (ii) magneto-rotational instabilities; (iii) interstellar medium stirring by dark matter substructure; or (iv) external gas accretion. The existence of the complex large-scale warping and asymmetries favors external gas accretion as one of the major energy sources that drives turbulence in the outer gas disk. We propose a scenario where this external accretion leads to turbulent adiabatic compression that enhances the turbulent velocity dispersion and might quench star formation in the outer gas disk of NGC 2683.
\end{abstract}

Key words. galaxies: individual: NGC 2683 - galaxies: ISM - galaxies: kinematics and dynamics

\section{Introduction}

The neutral gas content of a spiral galaxy can be roughly divided into three components: the dense molecular disk, the atomic gas disk, and an atomic gas halo. Whereas the inner gas disk is extremely thin $(\sim 100 \mathrm{pc}$; e.g. Kalberla \& Kerp 2009 for the Galaxy), the gas halo component, if present, is much more extended (a few kpc up to $22 \mathrm{kpc}$; Gentile et al 2013; Oosterloo et al. 2007) and contains about $15 \%$ of the galaxy's total gas content (NGC 891, Oosterloo et al. 2007; NGC 6946, Boomsma et al. 2008; NGC 253, Boomsma et al. 2005; NGC 4559, Barbieri et al. 2005; UGC 7321, Matthews \& Wood 2003; NGC 2403, Fraternali et al. 2002; NGC 2613, Chaves \& Irwin 2001; NGC 3198, Gentile et al. 2013; for a review see Sancisi et al. 2008). In edge-on systems, like NGC 891, the vertical extension of the halo gas is directly accessible.

The halo gas typically shows differential rotation parallel to the disk plane. Compared to the disk, the halo gas rotation velocity is lower (Fraternali et al. 2002; Oosterloo et al. 2007). In some cases, radial gas inflow is detected (Fraternali et al. 2002; Zschaechner et al. 2012). Above the star forming disk, the ionized component of the gas halo is detected in $\mathrm{H} \alpha$ (e.g.,

* Based on NRAO VLA observations (AI134). The National Radio Astronomy Observatory is a facility of the National Science Foundation operated under cooperative agreement by Associated Universities, Inc.

$\star \star$ The reduced data cube as a FITS file is only available at the CDS via anonymous ftp to cdsarc.u-strasbg. fr $(130.79 .128 .5)$ or via http://cdsarc.u-strasbg.fr/viz-bin/qcat?J/A+A/586/A98
Rossa \& Dettmar 2003), radio continuum (e.g., Dahlem et al. 2006), and X-rays (e.g., Yamasaki et al. 2009).

It is not clear what role the halo gas plays in the ecosystem of a spiral galaxy:

- Above the star forming disk, galactic fountains (Shapiro \& Field 1976; Fraternali \& Binney 2006) may play a dominant role. The disk gas is lifted into the halo by stellar winds and supernova explosions; then it cools there and finally falls back onto the galactic disk.

- The gas lifted by galactic fountains may interact with a preexisting hot ionized halo, leading to enhanced gas condensation and gas backfall.

- Accretion of intergalactic gas may add gas to the halo component. This accretion can be in form of the merging of small gas-rich satellites (van der Hulst \& Sancisi 2005).

The last scenario is corroborated by the detection of faint gaseous tidal streams in a considerable number of local spiral galaxies (Sancisi et al. 2008).

The HI disk of spiral galaxies is often warped and flares beyond the optical radius; i.e., its thickness increases exponentially. Flares are detected in the Galaxy (Kalberla \& Kerp 2009), M 31 (Brinks \& Burton 1984), and a sample of edge-on spiral galaxies (O'Brien et al. 2010; Zschaechner et al. 2012). While warps are ubiquitous in spiral galaxies where the HI disk is more extended than the optical disk (Garcia-Ruiz et al. 2002), flaring gas disks are common, but not ubiquitous: for modeling the HI observations of NGC 5746 (Rand \& Benjamin 2008) 
Table 1. Basic parameters of NGC 2683.

\begin{tabular}{lll}
\hline \hline Type & $\mathrm{Sb}$ & \\
\hline$m_{B}^{0}$ & $9.84 \mathrm{mag}$ & (de Vaucouleurs et al. 1976) \\
$D_{25}$ & $9.3^{\prime}=20.8 \mathrm{kpc}$ & (Nilson 1973) \\
Distance & $7.7 \mathrm{Mpc}$ & (Tonry et al. 2001) \\
$M_{B}$ & $-19.59 \mathrm{mag}$ & \\
$v_{\mathrm{rot}}^{\max }$ & $215 \mathrm{~km} \mathrm{~s}^{-1}$ & (Casertano \& van Gorkom 1991) \\
$\ddot{M}_{*}$ & $0.8 \mathrm{M}_{\odot} \mathrm{yr}^{-1}$ & (Irwin et al. 1999) \\
$l_{B}$ & $0.81^{\prime}=1.8 \mathrm{kpc}$ & (Kent 1985) \\
$l_{K^{\prime}}$ & $0.67^{\prime}=1.5 \mathrm{kpc}$ & (this paper) \\
$M_{*}$ & $3.6 \times 10^{10} M_{\odot}$ & (this paper) \\
$M_{*, \text { disk }}$ & $2.6 \times 10^{10} M_{\odot}$ & (this paper) \\
\hline
\end{tabular}

and NGC 4559 (Barbieri et al. 2005), a flaring gas disk is not necessary. According to van der Kruit (2007), the inner flat disk and the outer warped (and in some cases flared) one are distinct components with different formation histories. The inner disk forms initially, and the warped outer disk forms as a result of much later infall of gas with a higher angular momentum in a different orientation.

NGC 2683 is a good candidate for further investigating the role of the gas disk beyond the optical disk and gas halos for the evolution of a spiral galaxy. Its basic parameters are presented in Table 1. This highly inclined spiral galaxy is located at a distance of $7.7 \mathrm{Mpc}$. Within the galaxy a distance of $1 \mathrm{kpc}$ thus corresponds to $27^{\prime \prime}$. Deep VLA D array observations with a resolution of $\sim 1^{\prime}$ are ideal for probing its neutral gas halo. Casertano \& van Gorkom (1991) observed NGC 2683 for one hour with the VLA in D array configuration. They found neutral hydrogen extending more than twice as far as the visible light on both sides of the galaxy. The gas distribution is fairly symmetric and close to the plane of the optical disk. The derived rotation curve peaks at about $215 \mathrm{~km} \mathrm{~s}^{-1}$ at $\sim 3 \mathrm{kpc}$ from the galaxy center and then decreases monotonically. Kuzio de Naray et al. (2009) found a multi-valued, figure-of-eight velocity structure in the inner $45^{\prime \prime}=1.7 \mathrm{kpc}$ of the long-slit spectrum of NGC 2683 and twisted isovelocity contours in the velocity field. They argue that these features, along with boxy galaxy isophotes, are evidence of a bar in NGC 2683.

In this article we revisit NGC 2683, which we observed for nine hours in the HI $21 \mathrm{~cm}$ line with the VLA in D configuration. We also reduced archival $\mathrm{C}$ array observations and added them to our data. The observations are presented in Sect. 2, and the results are given in Sect. 3. The 3D kinematically modeling of the galaxy is described in Sect. 4 followed by the modeling results. We discuss our findings in Sect. 6 and give our conclusions in Sect. 7.

\section{Observations}

NGC 2683 was observed in December 2009 for nine hours with the Very Large Array (Napier et al. 1983) in D array configuration. We used $0542+498$ as flux calibrator. The phase calibrator $0909+428$ was observed for $2.5 \mathrm{~min}$ every $30 \mathrm{~min}$. The total bandwidth of $3.125 \mathrm{MHz}$ was divided into 128 channels with a channel separation of $5.16 \mathrm{~km} \mathrm{~s}^{-1}$. Of the 22 available antennas, 20 were EVLA antennas. To avoid closure errors on the VLAEVLA baselines, an alternative "channel 0" was created for the initial calibration stage. Subsequent calibration was achieved using VLA standard calibration procedures. Fifteen line-free channels were selected for continuum subtraction. The maps were generated with natural weighting to maximize sensitivity. We

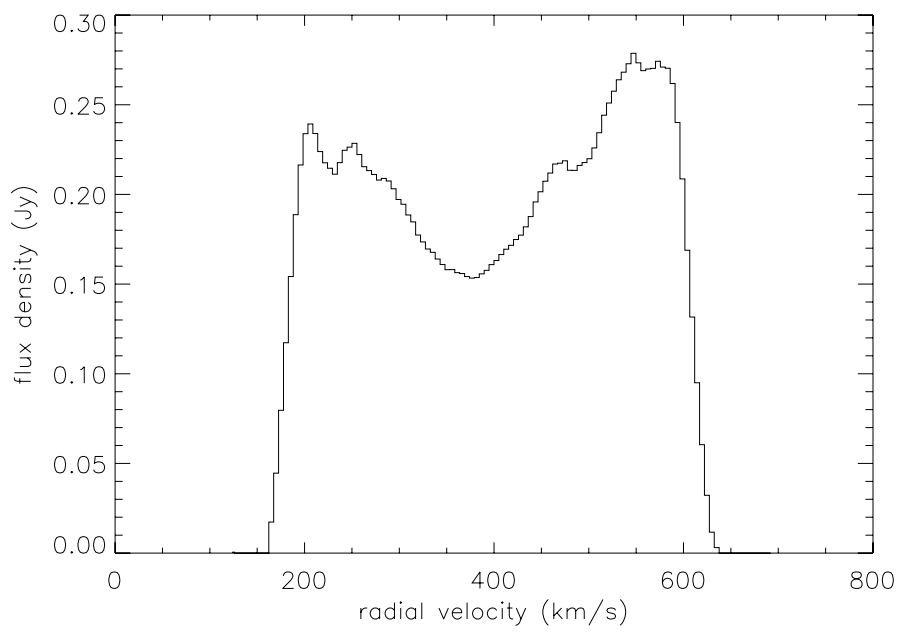

Fig. 1. Integrated HI spectrum of NGC 2683 from the VLA D array observations.

obtained an rms noise level of $1 \mathrm{mJy} /$ beam in a $5 \mathrm{~km} \mathrm{~s}^{-1}$ channel. The resolution is $61^{\prime \prime} \times 51^{\prime \prime}$.

In addition, we reduced $8.5 \mathrm{~h}$ of archival $\mathrm{C}$ array data. The total bandwidth of $3.125 \mathrm{MHz}$ was divided into 64 channels with a channel separation of $10.3 \mathrm{~km} \mathrm{~s}^{-1}$. Calibration was done using VLA standard calibration procedures. The maps were generated with a weighting between natural and uniform (ROBUST $=1$ ). An rms noise level of $0.4 \mathrm{mJy} / \mathrm{beam}$ in a $10.3 \mathrm{~km} \mathrm{~s}^{-1}$ channel was obtained with a resolution of $19^{\prime \prime} \times 18^{\prime \prime}$.

In a last step, the $C$ and $D$ array observations were combined into a single data set. With a ROBUST $=1$ weighting leading to a resolution of $21^{\prime \prime} \times 20^{\prime \prime}$, we obtained an rms noise level of $0.3 \mathrm{mJy} / \mathrm{beam}$ assuming a linewidth of $10.3 \mathrm{~km} \mathrm{~s}^{-1}$ channel. The cube was clipped at a flux density of $2.0 \mathrm{mJy} / \mathrm{beam}$ or 7 times the rms. Linear fits were made to the line-free channels of each spectrum. These baselines were then subtracted from the spectra. The HI column densities corresponding to the rms noise levels in individual channel maps are given in Table 2. For the creation of the moment 0 map, a boxcar smoothing of $7 \times 7 \times 7$ pixels $\left(49^{\prime \prime} \times 49^{\prime \prime} \times 36 \mathrm{~km} \mathrm{~s}^{-1}\right)$ was applied to the data cube and voxels of the smoothed cube with flux densities lower than $1 / 0.3 \mathrm{mJy} /$ beam for the $\mathrm{D} / \mathrm{C}+\mathrm{D}$ array data were blanked. The same voxels were then blanked in the original data cube before generating the moment 0 map. The primary beam correction was then applied to the moment 0 maps. At the end, the primary beam correction was applied to the channel maps. For the comparison with our models, we smoothed the $\mathrm{D}$ array data cube to the spectral resolution of the $\mathrm{C}+\mathrm{D}$ array data cube $\left(10.3 \mathrm{~km} \mathrm{~s}^{-1}\right)$.

We made continuum maps from the line-free channels of the HI data cube. However, owing to the small number of line-free channels, our $20 \mathrm{~cm}$ continuum image has a high rms noise level of $0.35 \mathrm{mJy} / \mathrm{beam}$ at a resolution of $60^{\prime \prime}$ compared to the rms noise level of $1 \mathrm{mJy} /$ beam in a $5.16 \mathrm{~km} \mathrm{~s}^{-1}$ channel (Table 2). On this image only the inner star forming disk of $\sim 4^{\prime}$ diameter is visible.

\section{Results}

We extracted an integrated spectrum from the area containing line emission (Fig. 1). The total line flux is $F_{\mathrm{HI}}=$ 101.4 $\mathrm{Jy} \mathrm{km} \mathrm{s}^{-1}$. This is coincidentally equal to the line flux that is corrected for self-absorption, but higher than the pointing/extension-corrected line flux of $F_{\mathrm{HI}}=87.6 \mathrm{Jy} \mathrm{km} \mathrm{s}{ }^{-1}$ from Springob et al. (2005). With a distance of $7.7 \mathrm{Mpc}$, this 
Table 2. HI observations and the combined data cube.

\begin{tabular}{llll}
\hline \hline Date & December 2009 & May 2004 & \\
\hline Array & $\mathrm{D}$ & $\mathrm{C}$ & $\mathrm{CD}$ \\
Duration & $9 \mathrm{~h}$ & $8.5 \mathrm{~h}$ & $17.5 \mathrm{~h}$ \\
Bandwidth & $3.125 \mathrm{MHz}$ & $3.125 \mathrm{MHz}$ & $3.125 \mathrm{MHz}$ \\
Number of channels & 128 & 64 & 64 \\
Central velocity $^{a}$ & $412 \mathrm{~km} \mathrm{~s}^{-1}$ & $412 \mathrm{~km} \mathrm{~s}^{-1}$ & $412 \mathrm{~km} \mathrm{~s}^{-1}$ \\
Velocity resolution $^{-1}$ & $5.16 \mathrm{~km} \mathrm{~s}^{-1}$ & $10.30 \mathrm{~km} \mathrm{~s}^{-1}$ & $10.30 \mathrm{~km} \mathrm{~s}^{-1}$ \\
FWHP restoring beam & $61^{\prime \prime} \times 51^{\prime \prime}$ & $19^{\prime \prime} \times 18^{\prime \prime}$ & $21^{\prime \prime} \times 20^{\prime \prime}$ \\
rms noise in channel maps & $1.0 \mathrm{mJy} / \mathrm{beam}^{\prime}$ & $0.4 \mathrm{mJy} / \mathrm{beam}^{19}$ & $0.3 \mathrm{mJy} / \mathrm{beam}^{-2}$ \\
Equivalent HI column density & $2 \times 10^{18} \mathrm{~cm}^{-2}$ & $1.3 \times 10^{19} \mathrm{~cm}^{-2}$ & $8 \times 10^{18} \mathrm{~cm}^{-2}$ \\
\hline
\end{tabular}

Notes. ${ }^{(a)}$ Heliocentric, optical definition.

corresponds to a total HI mass of $M_{\mathrm{HI}}=1.42 \times 10^{9} M_{\odot}$. The line flux of the $\mathrm{C}$ array data is $F_{\mathrm{HI}}=73.8 \mathrm{Jy} \mathrm{km} \mathrm{s}^{-1}$ corresponding to $M_{\mathrm{HI}}=1.03 \times 10^{9} M_{\odot}$. The integrated line profile is asymmetric with a $16 \%$ higher peak flux density on the receding side. The linewidths at $20 \%$ and $50 \%$ of the peak flux density are $W_{20}=450 \mathrm{~km} \mathrm{~s}^{-1}$ and $W_{50}=426 \mathrm{~km} \mathrm{~s}^{-1}$. With a systemic velocity of $415 \mathrm{~km} \mathrm{~s}^{-1}$ (Casertano \& van Gorkom 1991), the approaching and receding sides of the galactic disk contain equal amounts of atomic gas. The channel maps of the D array and combined $\mathrm{C}+\mathrm{D}$ array observations are presented in Appendix B.

\subsection{HI gas distribution}

The distribution of atomic hydrogen of NGC 2683 is presented in Fig. 2. As already shown by Casertano \& van Gorkom (1991), the atomic hydrogen is distributed over a diameter of $26.5^{\prime}$, so almost three times the optical diameter (Fig. 3). The overall structure is that of an inner disk of high column density gas $\left(\sim 9^{\prime}\right.$ diameter) and an extended low column-density vertically extended structure. We show that a part of this vertical extent is caused by the projection of a flaring gas disk. While the high column gas disk is thin, the low column density gas is more extended in the vertical direction.

The thin gas disk appears significantly bent. Whereas the southwestern part of the high column density gas disk at a distance of $3.5^{\prime}$ from the galaxy center bends away from the major axis to the south, the northeastern part bends slightly to the north. This warping is reversed at distances between $3.5^{\prime}$ and $8^{\prime}$ from the galaxy center, which means that at these distances the northeastern and southwestern parts bend toward the major axis. The projected disk thickness increases slightly with increasing distance from the galaxy center. At distances greater than $8^{\prime}$, the vertical projected thickness of the gas disk decreases rapidly. At the extremities of the gas disk, at distances of $\sim 11.5^{\prime}=26 \mathrm{kpc}$, two distinct gas blobs are present. The northeastern blob has a column density that is twice as high as the southwestern one. In the higher resolution $\mathrm{C}+\mathrm{D}$ array image, the southwestern part of the gas disk $(085220,+332200$, red circle in the right panel of Fig. 2) has a significant substructure. The gas mass within the depicted area is approximately $6 \times 10^{7} M_{\odot}$. The mass excess compared to the opposite side of the galactic disk (0852 55, +333000 , a second red circle in the right panel of Fig. 2) is about $1.5 \times 10^{7} M_{\odot}$.

\subsection{HI kinematics}

The velocity field of highly inclined galaxies cannot be used to derive the kinematical parameters (e.g.,
Kregel \& van der Kruit 2004) that have to be derived directly from the data cube. We derived the rotation curve of NGC 2683 from the position velocity diagram along the major axis and validated it with our 3D model (Sect. 4). To search for counterrotating gas and determine the rotation curve, we decreased the rms noise level in the position velocity diagrams by smoothing the velocity axis with a boxcar function of a width of three pixels or $15.5 \mathrm{~km} \mathrm{~s}^{-1}$. The position velocity diagram along the major axis from the D array data is presented in Fig. 4. We did not observe any counter-rotating gas. To derive the rotation curve from the position velocity diagram, we assumed that the neutral hydrogen moves in circular orbits and that the highest velocities trace the rotation curve at a given radius. The envelope-tracing method (Sofue \& Rubin 2001) makes use of the terminal velocity in a position velocity diagram along the major axis. The rotation velocity $v_{\text {rot }}$ is derived by using the terminal velocity $v_{\mathrm{t}}$ :

$v_{\text {rot }}=\left(v_{\mathrm{t}}-v_{\mathrm{sys}}\right) / \sin i-\Delta$,

where $\Delta=\left(\sigma_{\text {obs }}^{2}+\sigma_{\text {ISM }}^{2}\right)^{\frac{1}{2}}=11.3 \mathrm{~km} \mathrm{~s}^{-1}$ with $\sigma_{\text {ISM }}=10 \mathrm{~km} \mathrm{~s}^{-1}$ and $\sigma_{\mathrm{obs}}=5.16 \mathrm{~km} \mathrm{~s}^{-1}$, which are the velocity dispersion of the interstellar medium (ISM) and the velocity resolution of the observations, respectively.

We adopted a similar approach: the spectra along the major axis were decomposed into Gaussian profiles after clipping at a level of $2 \mathrm{mJy} / \mathrm{beam}$. The maximum/minimum peak velocities of the Gaussian decompositions were then adopted as rotation velocities on the approaching/receding side of the galaxy and corrected for an inclination of $83^{\circ}$ (see Sect. 4) ${ }^{1}$. Moreover, we set $\Delta=15.5 \mathrm{~km} \mathrm{~s}^{-1}$. The derived velocity curve (solid line in Fig. 4) is very similar to those obtained with the envelope-tracing method (dashed line in Fig. 4) and derived by Casertano \& van Gorkom (1991; Fig. 2).

Position velocity diagrams parallel to the minor axis (positions are shown in the upper panels of Fig. C.6) are presented in Figs. C.2 and C.3. Almost all position velocity diagrams are asymmetric. In the inner gas disk, emission of higher surface brightness is found toward positive/negative offsets at the receding/approaching side of the galactic disk (p6/p4). Low surface brightness emission of highest velocities is found toward positive offsets at the approaching side (pv4), whereas the envelope of the emission distribution at the receding side is quite symmetric (pv6). At intermediate radii of the receding side (pv7 and pv8) emission of highest surface brightness is found toward negative offsets. At intermediate radii of the approaching side (pv2

1 Up to 30 Gaussian components were fitted to reproduce the spectra. In the inner disk 15-25 components were needed whereas in the outer disk 3-10 components were sufficient to fit the HI spectra. 

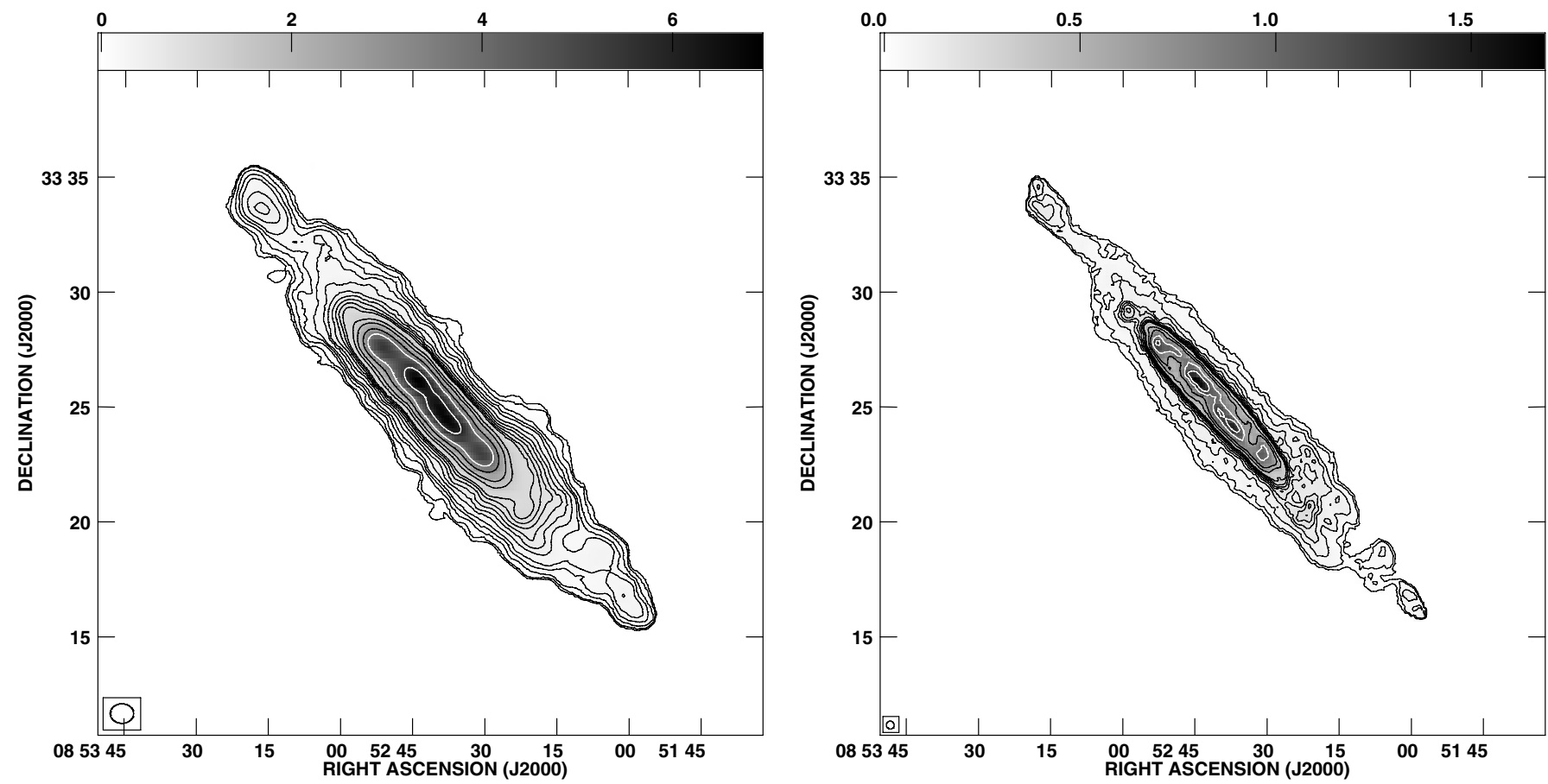

Fig. 2. HI gas distribution of NGC 2683. Left panel: from the D array observations. The beam size is $61^{\prime \prime} \times 51^{\prime \prime}$. The contour levels are $(1,2,4,6,8,12,16,20,24,28,32,48,64,96,128,192,264,392) \times 30 \mathrm{mJy} / \mathrm{beam} \mathrm{km} \mathrm{s}{ }^{-1}$ or $1.1 \times 10^{19} \mathrm{~cm}^{-2}$. The horizontal wedge is in units of Jy/beam $\mathrm{km} \mathrm{s}^{-1}$. Right panel: from the C+D array observations. The beam size is $21^{\prime \prime} \times 20^{\prime \prime}$. The contour levels are $(2,4,6,8,12,16,20,24,28,32,48,64,96,128,192,264,392) \times 10 \mathrm{mJy} / \mathrm{beam} \mathrm{km} \mathrm{s}{ }^{-1}$ or $2.6 \times 10^{19} \mathrm{~cm}^{-2}$. Grayscales are in units of Jy/beam km s${ }^{-1}$. As in Casertano \& van Gorkom (1991) we deliberately have suppressed the enhanced noise due to the primary beam correction (for comparison see Figs. C.2, C.3, B.3, and B.5).

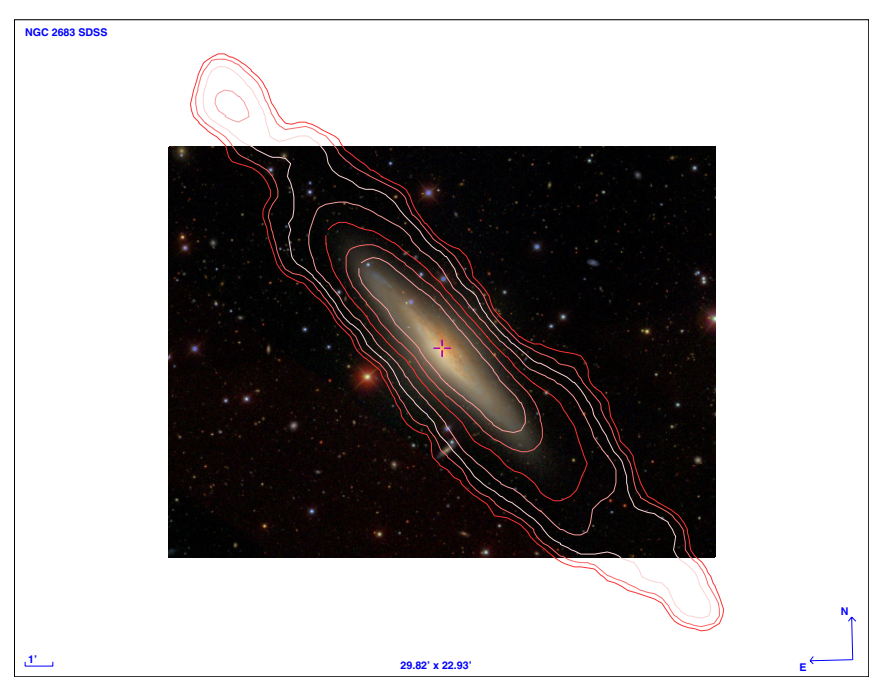

Fig. 3. HI contours on the SDSS color image of NGC 2683 () Michael R. Blanton \& David W. Hogg, 2006). The contour levels are $(2,4$, $8,16,32,64,128) \times 10 \mathrm{mJy} /$ beam $\mathrm{km} \mathrm{s}^{-1}$ or $2.6 \times 10^{19} \mathrm{~cm}^{-2}$.

and pv3) most emission is found toward positive offsets. In the outer part of the disk (pv1 and pv9), the emission is shifted toward positive offsets.

\section{3D modeling}

We developed an IDL code to construct 3D data cubes with properties identical to the observed data cubes ( $D$ and $C+D$ array data) from these model gas distributions. The sensitivity limits of our observations were also applied to the model cubes.

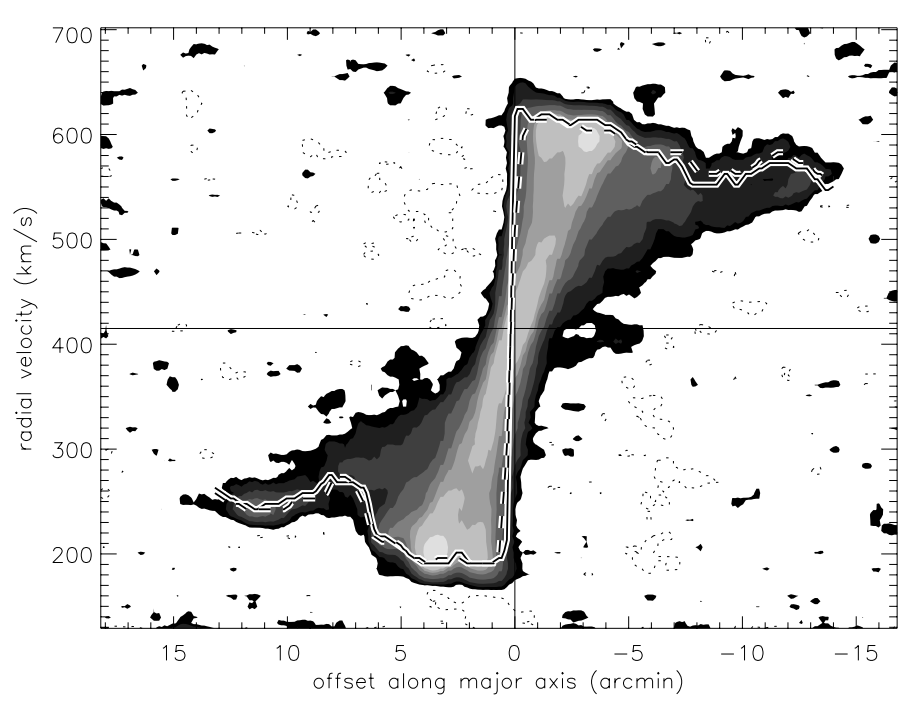

Fig. 4. Position velocity diagram along the major axis from the D array observations. At each position along the major axis, the highest velocities trace the rotation curve at that radius (solid line). The dashed line shows the rotation velocity derived with the envelope-tracing method. The grayscale levels are $(-1,1,2,4,8,16,24,32,64,132) \times$ $1 \mathrm{mJy} /$ beam.

For the 3D modeling we used a symmetrized mean rotation velocity (lower panel of Fig. 5), which we obtained by the Gaussian decomposition of the spectra along the major axis corrected by inclination (lower panel of Fig. 5). The rotation curve is consistent with the one derived by Casertano \& van Gorkom (1991). It is flat $\left(220 \mathrm{~km} \mathrm{~s}^{-1}\right)$ up to a galactic radius of $\sim 10 \mathrm{kpc}$. 

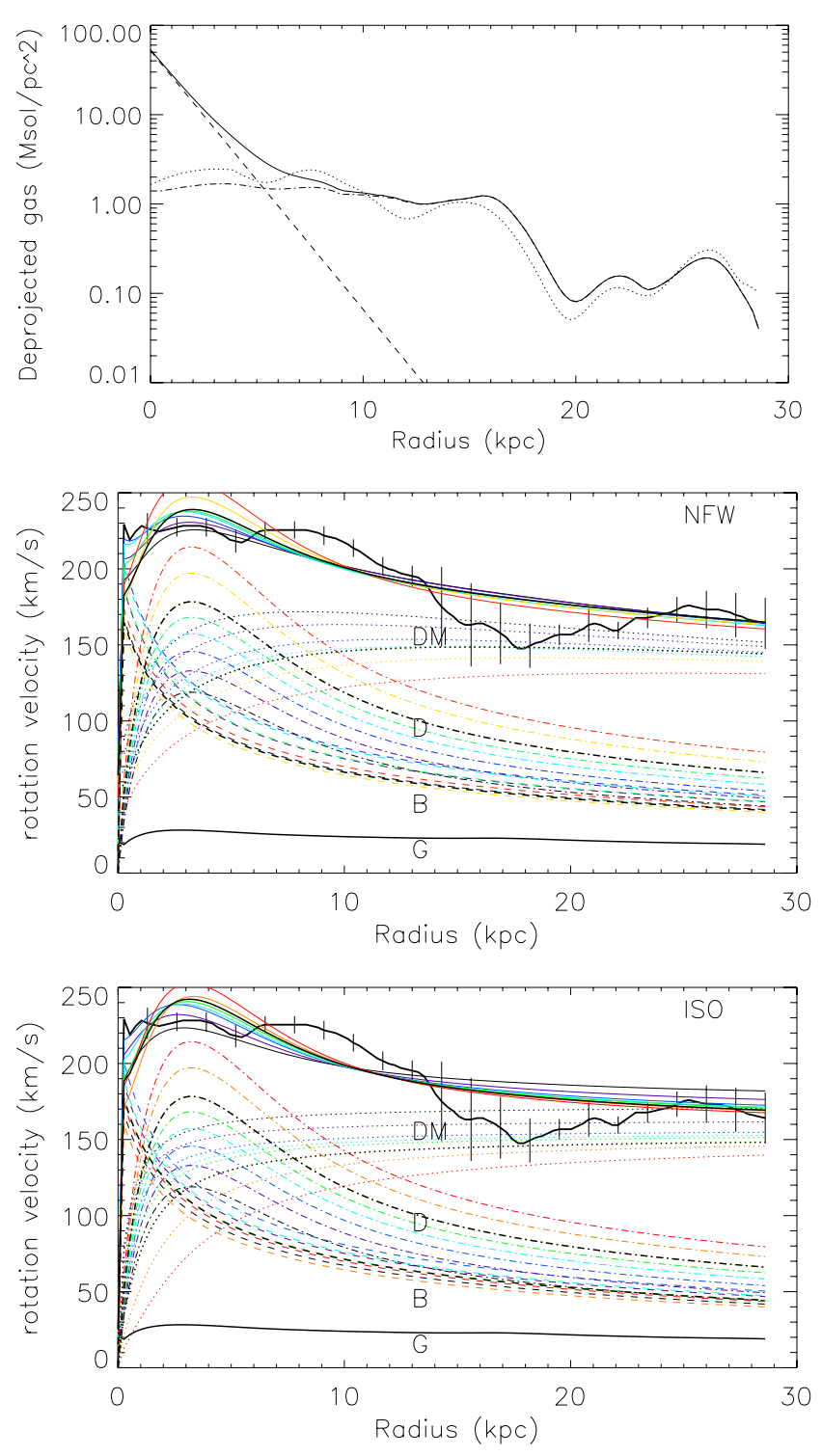

Fig. 5. Radial profiles of NGC 2683. Upper panel: deprojected total (solid), atomic (dash-dotted), and assumed molecular (dashed) gas surface density based on SEST CO-line observations (see Sect. 4). The dotted line represents the symmetric deprojected HI profile obtained from the method of Warmels (1988). Lower panel: observed and modeled rotation curve (solid), component due to the stellar bulge and disk (dashed), gas (dash-dotted), and dark matter halo (dotted). The colors indicate different $K^{\prime}$ band mass-to-light ratios from blue $(0.5)$ to red (1.3).

The rotation velocity declines to a value of $160 \mathrm{~km} \mathrm{~s}^{-1}$ at $18 \mathrm{kpc}$ and then rises to $200 \mathrm{~km} \mathrm{~s}^{-1}$ at a galactic radius of $26 \mathrm{kpc}$.

Following Warmels (1988), we derived the deprojected HI surface density using the symmetric rotation curve and the major axis position velocity diagram. First, the so-called strip integral was calculated by integrating the HI column density map perpendicularly to the major axis, as defined by the dynamical center and position angle of the stellar disk. The approaching and the receding sides of this strip integral are deprojected separately, using the iterative deconvolution method of Lucy (1974). The iteration process was halted at the point when

$\sqrt{\frac{\left(\Sigma_{\mathrm{HI}}-\Sigma_{\text {model }}\right)^{2}}{\Sigma_{\mathrm{HI}}^{2}}}<10^{-5}$, where $\Sigma_{\mathrm{HI}}$ and $\Sigma_{\text {model }}$ are the observed and modeled HI surface densities. The profiles of the approaching and receding sides were averaged to yield a mean radial HI profile which is presented in the upper panel of Fig. 5. The total HI mass of the model is consistent with our observations. As in most nearby spiral galaxies, the deprojected HI surface density is about constant within the optical radius (see, e.g., Leroy et al. 2008). Its value of $\sim 2 M_{\odot} \mathrm{pc}^{-2}$ is remarkably low compared to the typical value of $\sim 10 M_{\odot} \mathrm{pc}^{-2}$ in nearby spiral galaxies: in the sample of Leroy et al. (2008), only NGC 3351 and NGC 2841 show such low surface densities within the optical disk. The inner surface density profile shows two peaks corresponding to an inner and outer ring structure at radii of $3 \mathrm{kpc}$ and $8 \mathrm{kpc}$. For larger radii the surface density decreases monotonically until $20 \mathrm{kpc}$, before rising again toward a peak at $26 \mathrm{kpc}$. We interpret and model the latter maximum as an $\mathrm{HI}$ ring at the extremity of the gas disk.

The gas surface density is the sum of the atomic and molecular gas surface densities when assuming a Galactic $\mathrm{CO}-\mathrm{H}_{2}$ conversion factor (upper panel of Fig. 5). For the molecular gas surface density, we used the SEST CO(1-0) line flux of Elfhag et al. (1996) and assumed an exponential distribution with a scale length of the stellar disk $40^{\prime \prime}=1.5 \mathrm{kpc}$, as observed in local spiral galaxies (Leroy et al. 2008). This led to a central surface density of molecular gas of $40 M_{\odot} \mathrm{pc}^{-2}$ (lower panel of Fig. 5). The atomic-to-molecular mass ratio of NGC 2683 is about 5. This ratio is located at the lower end of the atomic-to-molecular mass ratio distribution for $\mathrm{Sb}$ galaxies (Fig. 4 of Young \& Scoville 1991).

For the mass modeling of NGC 2683, we applied GALFIT (Peng et al. 2002) to the 2MASS K' image to obtain surface brightness profiles for the bulge and disk components of NGC 2683. The derived parameters (central surface density $\Sigma_{0}$, surface density at the effective radius of the Sersic profile ${ }^{2} \Sigma_{\mathrm{e}}$, scale length $l$, effective radius $R_{\mathrm{e}}$ of the Sersic profile, Sersic index $n$, and total stellar mass $M_{*}$ assuming a $K^{\prime}$ band mass-tolight ratio of 1.0) are given in Table 3 . The $K^{\prime}$ band stellar disk scale length is $l_{*}=1.5 \mathrm{kpc}$, a low value for a rotation velocity similar to that of the Galaxy or galaxies of comparable rotation velocities (Fig. 8 of Courteau et al. 2007). The decomposition of the rotation curve for different $K^{\prime}$ band mass-to-light ratios from 0.5 to 1.3 is shown in the lower panel of Fig. 5. Whereas the fit of the exponential disk is robust, the fit of the bulge component depends somewhat on the initial conditions that were used in GALFIT. After inspecting the residual image, we are confident that the mass profile is reproduced within the given errors (Table 3).

We assumed that the dark matter halo is isothermal with a core following the density distribution:

$\rho(R)=\frac{\rho_{0}}{1+\left(R / R_{0}\right)^{2}}$,

where $\rho_{0}$ is the central density and $R_{0}$ the core radius. Alternatively, we used an NFW profile (Navarro et al. 1996):

$\rho(R)=\frac{\rho_{0}}{\frac{R}{R_{\mathrm{S}}}\left(1+\frac{R}{R_{\mathrm{S}}}\right)^{2}}$,

where $\rho_{0}$ is the central density and $R_{\mathrm{S}}$ the scale radius. For a given $K^{\prime}$ band mass-to-light ratio $M / L_{K^{\prime}}$ for the bulge and the disk, the halo central density and core radius were determined by minimizing $\chi^{2}$ between the model and observed rotation curves.

2 The Sersic profile is given by $\Sigma=\Sigma_{\mathrm{e}} \exp \left(-\kappa\left(\left(R / R_{\mathrm{e}}\right)^{1 / n}-1\right)\right)$ with $\kappa=2 n-0.331$. 
Table 3. GALFIT derived model parameters of NGC 2683.

\begin{tabular}{llllllc}
\hline \hline & $M / L_{K^{\prime}}$ & $\begin{array}{l}\Sigma_{\mathrm{e}} / \Sigma_{0} \\
\left(M_{\odot} \mathrm{pc}^{-2}\right)\end{array}$ & $\begin{array}{l}R_{\mathrm{e}} / l \\
(\mathrm{kpc})\end{array}$ & $n$ & $\begin{array}{l}M_{*} \\
\left(10^{10} M_{\odot}\right)\end{array}$ & Axis ratio \\
\hline Bulge & 1.4 & $184 \pm 4$ & $1.68 \pm 0.07$ & $5.20 \pm 0.09$ & $1.20 \pm 0.02$ & 0.7 \\
Disk & 0.9 & $1825 \pm 43$ & $1.50 \pm 0.01$ & 1.0 & $2.58 \pm 0.05$ & - \\
\hline
\end{tabular}

We calculated $\chi^{2}$ for a grid of $\left(M / L_{K^{\prime}}\right)_{\text {bulge }},(M / L)_{\text {disk }}, \rho_{0}$, and $R_{0}$ yielding:

- for the cored isothermal halo $\left(\chi^{2}=3.10\right):\left(M / L_{K^{\prime}}\right)_{\text {bulge }}=$ $1.4,\left(M / L_{K^{\prime}}\right)_{\mathrm{disk}}=0.9, \rho_{0}=0.16 M_{\odot} \mathrm{pc}^{-3}$ and $R_{0}=2.0 \mathrm{kpc}$; and

- for a NFW halo $\left(\chi^{2}=3.63\right):\left(M / L_{K^{\prime}}\right)_{\text {bulge }}=1.2$, $\left(M / L_{K^{\prime}}\right)_{\text {disk }}=0.9, \rho_{0}=0.03 M_{\odot} \mathrm{pc}^{-3}$ and $R_{0}=7.5 \mathrm{kpc}$.

Based on the $\chi^{2}$ values, the cored isothermal halo is the preferred model.

For our modeling, we adopted a mass-to-light ratio of $M / L_{K^{\prime}}=1.4$ for the bulge and $M / L_{K^{\prime}}=0.9$ for the disk component. The latter value is compatible with the value of 0.8 given by Bell et al. (2003; Appendix B). However, it is situated at the very high end of the $M / L_{K^{\prime}}$ distribution determined by the DiskMass Survey (Fig. 1 of Martinsson et al. 2013). Lower values of the mass-to-light ratio lead to gas velocity dispersions lower than typically observed in the inner disks of nearby spiral galaxies (Tamburro et al. 2009; see Sect. 6.3).

The GALFIT absolute magnitude of $M_{K_{\mathrm{s}}}=6.328 \pm 0.017$ is consistent with the value of $M_{K_{\mathrm{s}}}=6.30 \pm 0.01$ from the 2MASX catalog (Skrutskie et al. 2006). The disk/bulge decomposition gave disk and bulge masses of $2.6 \times 10^{10} M_{\odot}$ and $1.2 \times 10^{10} M_{\odot}$, respectively. We thus determined a total stellar mass of $M_{*}=3.8 \times 10^{10} M_{\odot}$. This is quite low for a rotation velocity of $220 \mathrm{~km} \mathrm{~s}^{-1}$ (Fig. 8 of Courteau et al. 2007).

To investigate the 3D structure of the atomic gas disk, we made different 3D axisymmetric models for which we produced model HI data cubes. These models have the following components:

- a thin gas disk with a thickness of 500 pc (TD);

- a radially decreasing velocity dispersion $(\mathrm{Vd})$;

- an asymmetric disk structure (E);

- a possible warp in inclination and position angle of the thin disk (PA, INC);

- different gas flares at galactic radii larger than $9 \mathrm{kpc}(\mathrm{F} 0-\mathrm{F} 3$; Fig. 9);

- an outer gas ring $(R>25 \mathrm{kpc})$;

- a gas halo around the inner disk $(\mathrm{H})$; and

- a vertically lagging rotation velocity (L).

For the inclination angle of the galactic disk, we set $90^{\circ} \leq i \leq$ $60^{\circ}$. The comparison of the model position velocity diagrams with those of the high-resolution $\mathrm{C}+\mathrm{D}$ array data showed that $i=83^{\circ}$ is the best choice for the inner high surface density disk. In addition, it turned out that $i=87^{\circ}$ for the outer HI ring $(R>24 \mathrm{kpc})$ led to the best resemblance between the model and observed gas distributions (column TD in Fig. A.2). For galactic radii between 10 and $24 \mathrm{kpc}$, we considered warps in inclination and position angle.

To reproduce the emission distribution in the $\mathrm{D}$ array channel maps close to the systemic velocity (Fig. B.4), we introduced a turbulent velocity dispersion that increases toward the galaxy center:

$v_{\text {turb }}=10+10 \exp \left(-(R / 4 \mathrm{kpc})^{2}\right) \mathrm{km} \mathrm{s}^{-1}$.
For the flare, we assumed four characteristic radial profiles of the disk height $H=F W H M / 2$ (Fig. 9):

$\rho(z)=\Sigma_{0}(R) \operatorname{sech}(1.3 z / H(R))$,

where $\Sigma_{0}(R)$ is the surface density in the midplane. We also tried an exponential and $\operatorname{a~sech}^{2}$ (isothermal) dependence of the vertical density structure (see Sect. 4.3).

For radii larger than $R=10.5^{\prime}=23.5 \mathrm{kpc}$, which is the low surface density ring, the disk plane is shifted vertically by $35^{\prime \prime}=1.3 \mathrm{kpc}$. This was necessary because the two HI emission blobs at the extremities of the galactic gas disk are offset from the galaxy's major axis (see Sect. 3.1).

\subsection{Asymmetric structure of the disk}

Since the observed asymmetries in the position velocity diagrams (Sect. 3.2) cannot be reproduced with an axisymmetric model, we modeled the inner gas disk with elliptical isodensity annuli. This choice is motivated by the existence of another non-axisymmetric structure in NGC 2683, a galactic bar within $R=2 \mathrm{kpc}=54^{\prime \prime}$ (Kuzio de Naray et al. 2009). Since the observed HI asymmetries extend over more than $6^{\prime}=13 \mathrm{kpc}$ in radius, a physical link between the inner bar structure and outer elliptical annuli is not obvious, though.

The size of the major axis of the elliptical component is $a=15 \mathrm{kpc}$ and the axis ratio is $b / a=2 / 3$. We varied the angle between the major axis and the plane of the sky up to $90^{\circ}$ in steps of $10^{\circ}$. It turned out that an angle of $30^{\circ}$ reproduced our observations in a satisfying way (column TD+E in Figs. A.1 and A.2). This is similar to the result of Kuzio de Naray et al. (2009), who found the bar to be closer to side-on than end-on. The approximate alignment between the two structures might occur by chance or indicate a common pattern speed of the bar and the outer non-axisymmetric gas structure, i.e. a dynamical coupling between the two structures.

The resulting face-on projection of the gas distribution is presented in Fig. 6. The resulting surface brightness profile (dash-dotted line in the upper panel of Fig. 5) does not differ significantly from the initially symmetric deprojected profile. The elliptical structure of the inner gas disk might be interpreted as a crude approximation to spiral and/or bar structure (for more sophisticated models see Kamphuis et al. 2013). This corresponds to an $m=2$ distortion that can be measured by a Fourier decomposition of the HI distribution (e.g., Bournaud et al. 2005). Compared to the THINGS sample (Walter et al. 2008), NGC 2683 resembles (i) the irregular galaxy NGC 4214, which shows an inner bar-like HI structure within a symmetric disk; (ii) the Sab galaxy NGC 4736, which shows an inner HI ring with a different position angle than the main disk; and (iii) the Sc galaxy NGC 628, which shows an inner bar structure mainly in CO. If the elliptical structure is mainly due to a bar, we expect a distorted velocity field, i.e. the $3 \mathrm{D}$ velocity vectors are approximately tangential to the isophotes of the bar (Pérez et al. 2004). If it is mainly due to spiral arms, we expect a much less distorted velocity field. 


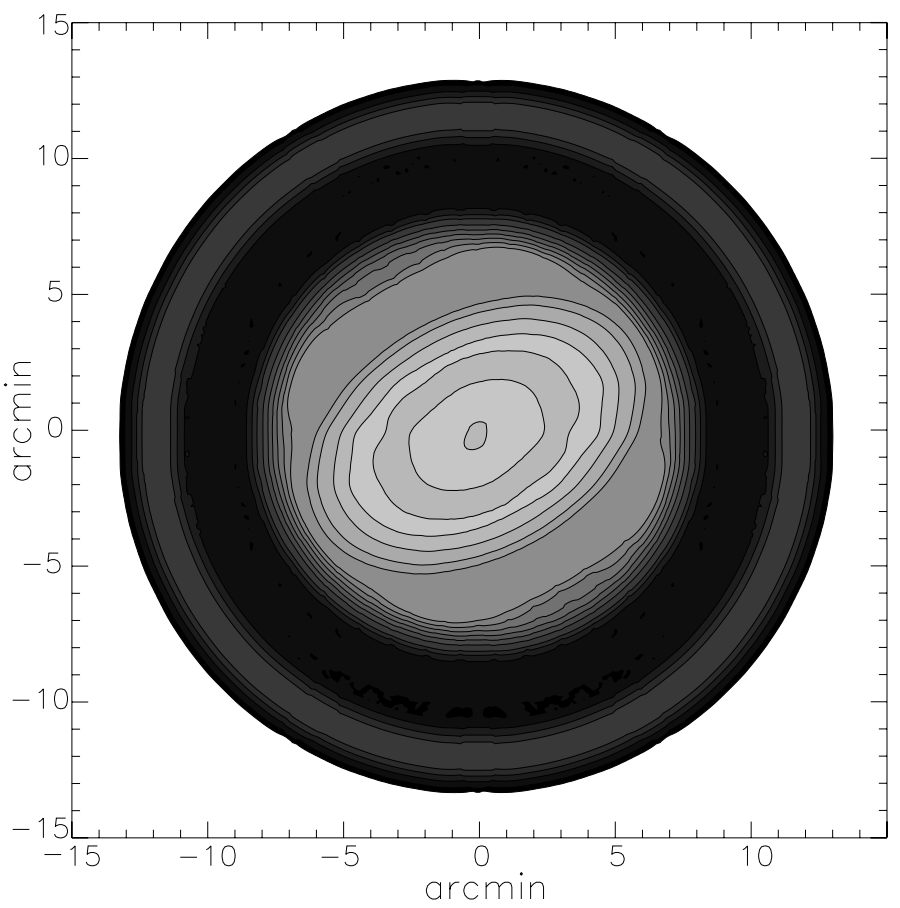

Fig. 6. Model gas distribution in face-on projection. The resolution is $61^{\prime \prime} \times 51^{\prime \prime}$. Grayscale levels are $(1,2,4,6,8,12,16,20,24,28,32,48$, $64,96,128,192,264,392,784) \times 5 \mathrm{mJy} /$ beam $\mathrm{km} \mathrm{s}^{-1}$ or $2 \times 10^{18} \mathrm{~cm}^{-2}$.

The influence of velocity distorsions on our model is shown in Fig. 7 where the position velocity diagrams of a disk model with an elliptical component and a velocity field following the bar isophotes is shown for different ellipticities and different angles between the bar's major axis and the plane of the sky. While including an asymmetric velocity field improves the resemblance of pv3, it worsens the resemblance of pv5, pv6, and pv7 to observations. Small velocity distortions are present in the inner disk of NGC 2683, because we observe an asymmetric position velocity diagram in the center of NGC 2683 (pv 5). However, the observed amplitude of the asymmetry is much smaller than that of the models. We thus conclude that the velocity field of the elliptical component is rather symmetric.

\subsection{The disk warp}

Can the projected thickness of the HI disk be caused by a warp of the thin disk? To answer this question, we chose five characteristic inclination and four position angle profiles to create tilted ring models of the thin HI disk (Fig. 8). The radial extent of the warp is set by the fact that warps typically begin at an HI column density of $\sim 2 M_{\odot} \mathrm{pc}^{-2}$ where the HI density drops down (GarciaRuiz et al. 2002). The maximum inclination/position angles of the model profiles were determined by the observed projected disk thickness. We created model cubes for all combinations of the inclination and position angle profiles. For clarity, we only show selected $\mathrm{C}+\mathrm{D}$ array position velocity diagrams of nine of these combinations in Fig. C.1.

In the two position velocity diagrams of the approaching side (pv3 and pv4), the emission at negative offsets is reproduced well by the models. However, the linewidth of the emission at positive offsets is significantly smaller than the observed linewidth. On the receding side (pv6 and pv7), we observe the opposite trend: the linewidth of the emission at negative offsets is significantly smaller than the observed linewidth. In addition, the linewidth at offsets $>1^{\prime}$ are also smaller than observed. These differences between our models and observations are present for all 20 models. We thus conclude that the projected thickness of the HI disk cannot be reproduced by a warp of the thin disk alone.

To reproduce the slight warp of at least a part of the gas disk between $7 \mathrm{kpc}$ and $14 \mathrm{kpc}$ observed in the high and low resolution HI distribution maps (Fig. 2), we selected a best-fit warp model by eye based on the moment 0 maps and position velocity diagrams (Fig. 8). By only varying the position angle, we did not succeed in finding an axisymmetric model that reproduced the HI gas distribution in a satisfying way. Without a varying inclination angle, the spatial extent of the position velocity diagrams pv3, pv4, pc6, and pv7 at velocities close to the systemic velocity is too small (Figs. C.2 and C.3). Varying the inclination by $10^{\circ}$ toward lower inclination angles improved the observed position velocity diagrams in a satisfying way (column $\mathrm{TD}+\mathrm{E}$ compared to TD+E+INC of Figs. A.1 and A.2). However, the spatial extent of the position velocity diagrams, especially beyond the optical disk (pv1, pv2, pv3, pv7, pv8, pv9), are significantly smaller than our observations.

Thus, the HI data is consistent with a thin disk component of constant position angle and a change in inclination by $10^{\circ}$ between $10 \mathrm{kpc}$ and $23 \mathrm{kpc}$. A second thick component is needed beyond the optical disk. The outer low surface brightness ring is modeled best with an inclination of $87^{\circ}$.

\subsection{The disk flare}

As shown in the previous section, the observed position velocity diagrams beyond the optical radius cannot be reproduced by a tilted ring model that only includes a thin disk (columns TD in Figs. A.1 and A.2). We therefore introduced a flaring gas disk into our model. The disk flare was modeled by an exponential rise in the disk height $H(R)=F W H M / 2$. The maximum disk width FWHM is set by the comparison between (i) the outer contours of the model and observed HI moment0 maps and (ii) the $\mathrm{D}$ array position velocity diagrams beyond the optical radius: $F W H M=2.6 \mathrm{kpc}$. We varied the radial scale length of the exponential rise of $H(r)$ between $9 \mathrm{kpc}$ and $2 \mathrm{kpc}$. Moreover, we assumed that the flare saturates at a width of $4 \mathrm{kpc}$ and might decrease at larger radii. The four different types of warps that we tested in our model (F0-F3) are shown in Fig. 9.

For the vertical distribution of the atomic gas density, we additionally tested an exponential and a $\operatorname{sech}^{2}$ profile (Fig. A.3). We adapted the respective scale heights to obtain the same FWHM for all profiles. Whereas the $\operatorname{sech}^{2}$ profile is indistinguishable from the sech profile, the exponential vertical profile broadens the position velocity diagrams in the velocity direction. Since this broadening is consistent with most of the observed position velocity diagrams, an exponential vertical distribution might be the better choice. Keeping this in mind, we decided to keep the vertical sech profile of Eq. (6) which is in between the isothermal $\left(\operatorname{sech}^{2}\right)$ and the exponential case.

\subsection{Rotation velocity lag}

Thick HI components with lagging rotation velocities are observed in NGC 891 (Oosterloo et al. 2007) and NGC 3198 (Gentile et al. 2013), among others. While Oosterloo et al. (2007) modeled the thick component separately by assigning it a lower rotation velocity, Gentile et al. (2013) assumed a constant vertical gradient. We assumed a linear vertical gradient of the 


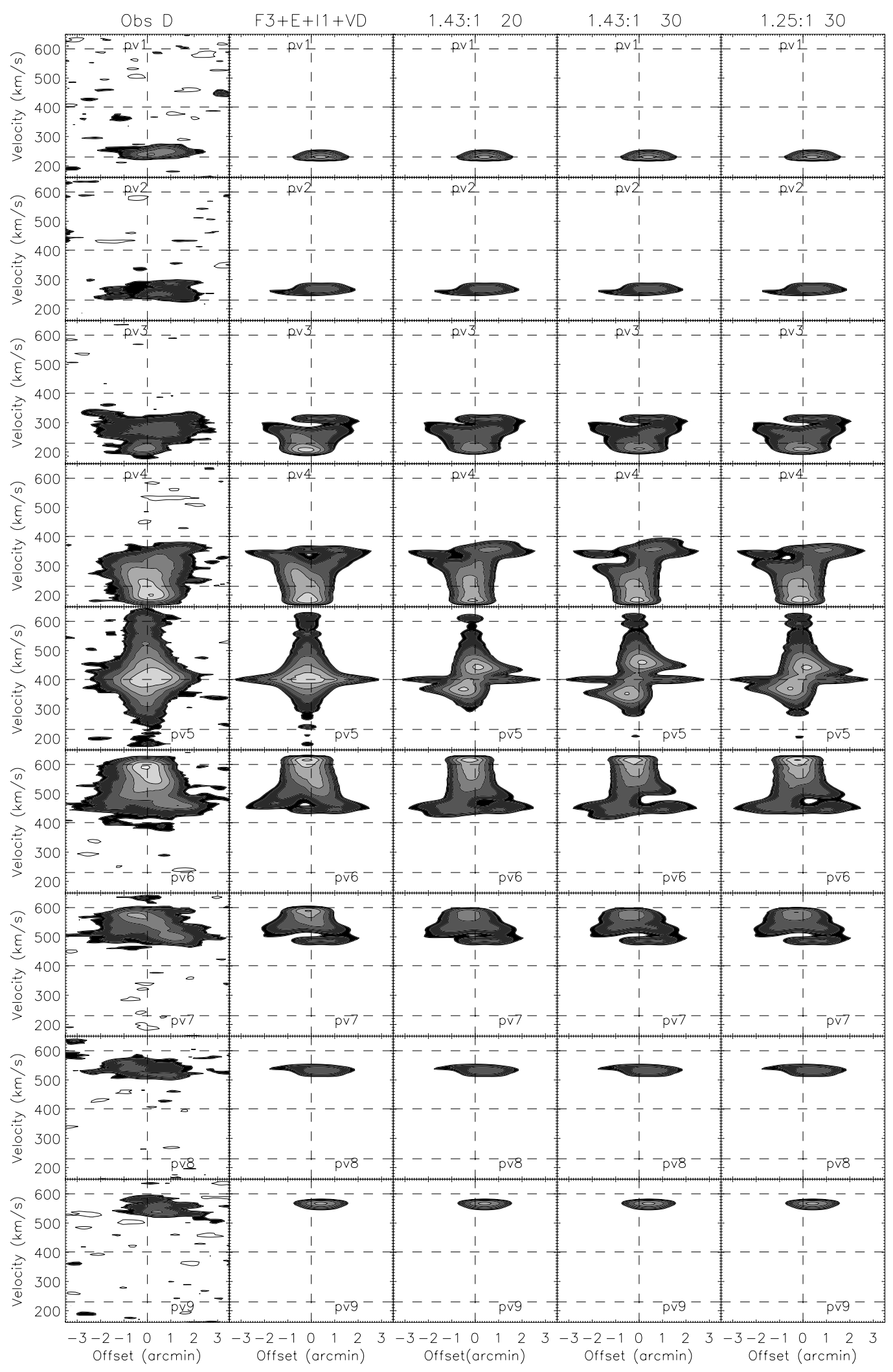

Fig. 7. NGC $2683 \mathrm{HI}$ D array and model position velocity diagrams. The contour levels are $(-2,2,3,6,12,24,48,96) \times 0.7 \mathrm{mJy} / \mathrm{beam}$. The resolution is $61^{\prime \prime} \times 51^{\prime \prime}$. The model includes an elliptical component with axis ratios of 1.43:1/1.25:1 and angles between the bar's major axis and the plane of the sky of $20^{\circ}$ and $30^{\circ}$, a warp in inclination (I1), a centrally increasing velocity dispersion (VD), and a disk flare (F3).

following form:

$v_{\text {rot }}(z)=v_{\text {rot }, 0}-\xi\left(\frac{z}{1.0 \mathrm{kpc}}\right)$,

A98, page 8 of 29 where $v_{\text {rot, } 0}$ is the axisymmetric rotation velocity and $\xi=$ $5,10,15 \mathrm{~km} \mathrm{~s}^{-1}$. These gradients are comparable to those found (7) in NGC 891 and NGC $3198\left(\Delta v / \Delta z \sim 7-15 \mathrm{~km} \mathrm{~s}^{-1} \mathrm{kpc}^{-1}\right)$. 

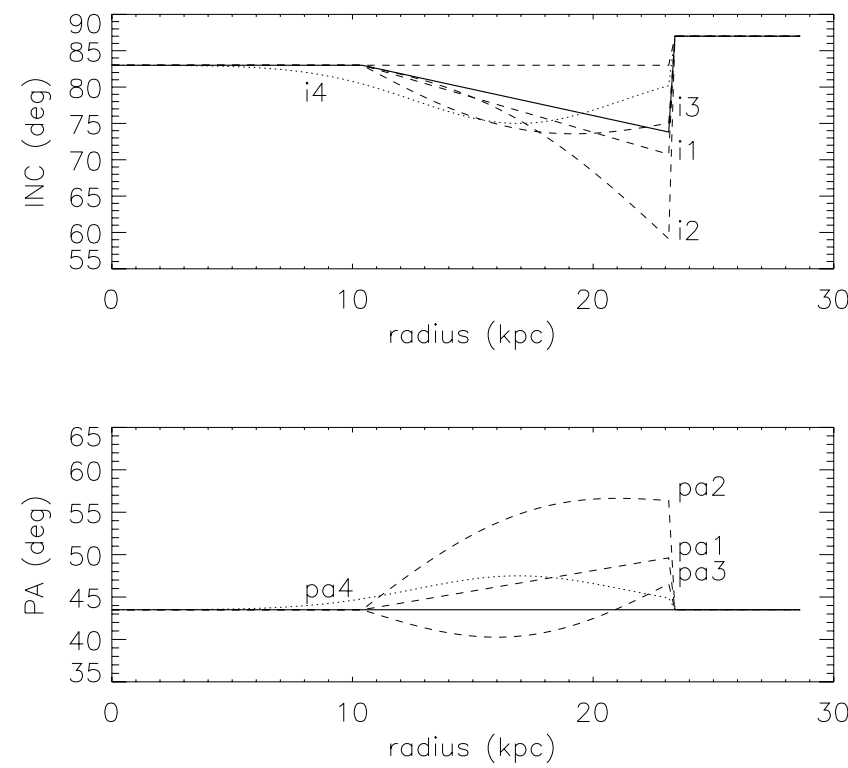

Fig. 8. Model inclination and position angle with respect to the galactic radius. The best-fit parameters are shown with a solid line. The dashed and dotted lines represent the tested variations for discarded models. The model with profiles represented by dotted lines are not shown in Fig. C.1.

Since we are interested in a lagging HI halo, different HI halos with the different velocity lags are presented in Fig. C.7 (for the halo component, we refer to Sect. 5.5). The velocity lag makes the radial velocities of gas at high altitudes (or offsets) increase/decrease toward the systemic velocity (see also Sect. 5.5).

\section{Comparison with observations}

For the following comparison between models and observations, we started with an inclined thin disk model and then successively added a decreasing velocity dispersion $(\mathrm{Vd})$, an elliptical component (E), a warp in position angle (PA) and inclination angle (INC), the different flare profiles (F0-F3), a rotation velocity lag (L), and an HI halo $(\mathrm{H})$. For the models including a disk flare, only the best-fit warp of Sect. 4.2 was considered. The gas distribution maps (Fig. C.6) and nine position velocity diagrams along the galaxy's minor axis were then calculated in the same way as for the observations (Figs. C.2, C.3, B.4, and B.6).

\subsection{Position velocity diagrams}

The addition of the elliptical disk component is necessary to reproduce the asymmetric emission distribution in the position velocity diagrams of the inner gas disk (pv4, pv6, and pv7; compare column TD with column TD+E of Figs. A.1 and A.2). The addition of a warp along the line of sight leads to an increased spatial extent of the V-shaped emission distribution (with two "legs") at velocities close to the systemic velocity in the position velocity diagram (the legs of the $\mathrm{V}$ are curved outwards). This leads to a closer resemblance between the model and observed position velocity diagrams of the inner disk mostly on the receding side (pv6 and pv7; compare column TD+E with column TD+E+i1 of Figs. A.1 and A.2). On the approaching side, the warp model is consistent with the observed position velocity diagram pv3. However, the increased spatial extent of the V-shaped emission distribution is not observed in pv4.

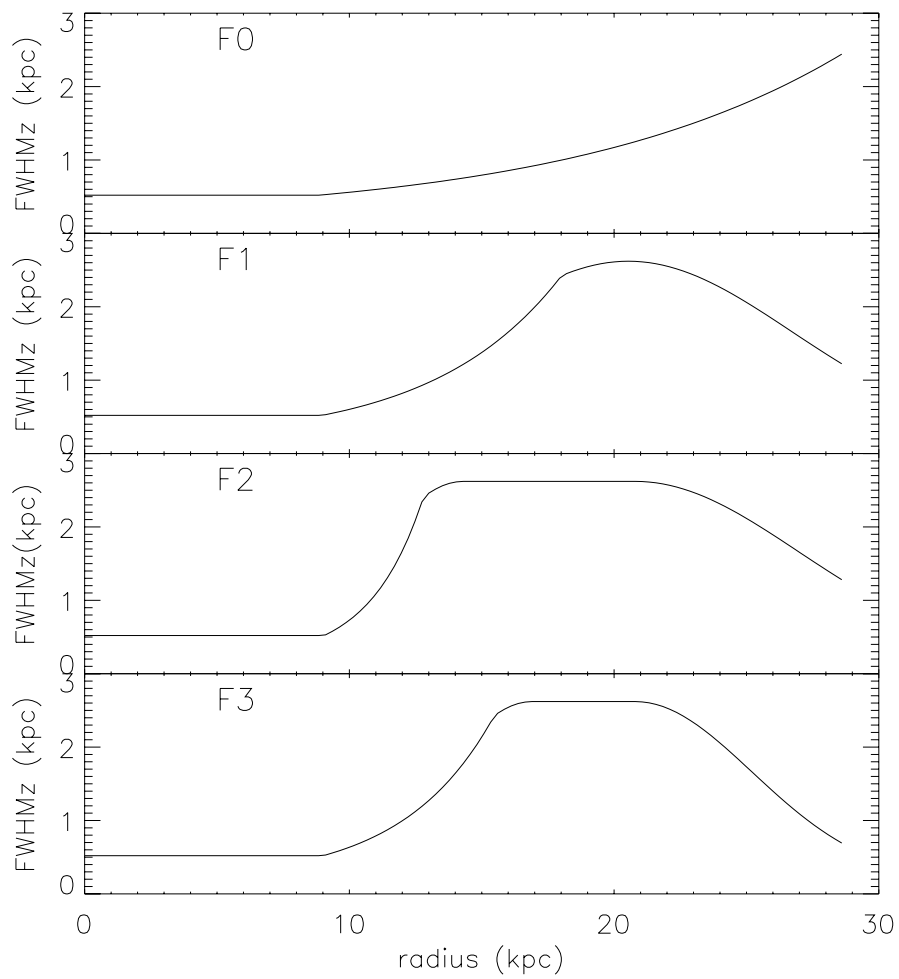

Fig. 9. Model disk flares.

The different flare models lead to different spatial extents of the emission in the position velocity diagrams at different galactic radii (flares F0-F3 in Fig. C.2 (D array) and C.3 (C+D array)). We discuss these differences from negative ( $\mathrm{pv} 1)$ to positive offsets (pv9). If not stated otherwise, the discussed features are present in the $\mathrm{D}$ and $\mathrm{C}+\mathrm{D}$ array data.

$p v 1$ : Model F0/F3 shows the largest/smallest spatial extent of the HI emission, comparable to or somewhat smaller than the observed extent. The model flares F0, F1, and F2 resemble the observed emission distribution most closely.

$p v 2$ : Models F0-F2 show the largest spatial extents of the high column density HI emission, somewhat larger than the observed extent. The observed low column density extension to negative offsets (D array data) is not present in model F0. Model F3 resembles the observed emission distribution most closely.

pv3: the observed emission distribution is not reproduced well by the flare models. The legs of the $\mathrm{V}$-shaped structure are too thin for the model flares F0 and F1 (C+D array data). The spatial extent of the observed emission distribution is small at low velocities, but significantly larger for the model F2 $(\mathrm{C}+\mathrm{D}$ array data). The model F3 represents a compromise that is marginally consistent with observations.

$p v 4$ : as for $\mathrm{pv} 3$, the legs of the $\mathrm{V}$-shaped emission distribution are too thin for the model flares F0/F1 and too thick for the model F2 in the $\mathrm{C}+\mathrm{D}$ array data. This effect is less obvious in the D array data. The model F3 resembles the observed emission distribution most closely.

$p v 5$ : the observed asymmetric emission is not reproduced by our models.

pv6: as for $\mathrm{pv} 3$, the legs of the $\mathrm{V}$-shaped emission distribution are too thin for the model flares F0/F1. The width of the leg at negative offsets is reproduced better by model F2, and the leg at positive offsets by model F3. This is most visible in the $\mathrm{C}+\mathrm{D}$ data. 
$p v 7$ : the spatial extent of the observed emission distribution is small at low velocities and significantly larger for model F2. The legs of the V-shaped emission structures of the models flares F0 and $\mathrm{F} 1$ are thinner than the corresponding observed structures (most visible in the $\mathrm{C}+\mathrm{D}$ array data). Model flare $\mathrm{F} 3$ represents a compromise that is most consistent with observations.

$p v 8$ : the observed high column density part of the emission distribution is located at somewhat higher velocities than the low column density part. This feature is not reproduced by our models. The observed extent of the low column density emission to negative offsets ( $\mathrm{D}$ array data) is not present in model F0. Models F1-F3 approximately reproduce the observed emission distribution.

pv9: the spatial extent of the emission distribution of models F0, $\mathrm{F} 1$, and F2 are larger than observed. Only model F3 reproduces the observed extent.

We conclude that model F3 is most consistent with our HI observations.

\subsection{Selected channel maps}

Selected channel maps of the different models and the HI observations are shown in Fig. C.4 for the D array and in Fig. C.5 for the $\mathrm{C}+\mathrm{D}$ array data. A decreasing inclination angle leads to an increasing angle of the horizontal $\mathrm{V}$-shaped emission structure (the $\mathrm{V}$ becomes wider) in the channel maps. This is visible the most in the $\mathrm{C}+\mathrm{D}$ data between 300 and $506 \mathrm{~km} \mathrm{~s}^{-1}$ (Fig. B.2). The model with a varying inclination angle $(\mathrm{TD}+\mathrm{E}+\mathrm{INC})$ reproduces observations better than the model with a constant inclination angle (TD+E).

The addition of a flare to the model leads to an increased extent for the emission distribution in the vertical direction. This effect is visible best in the channel maps between 299 and $508 \mathrm{~km} \mathrm{~s}^{-1}$ (Fig. C.4). The legs of the horizontal V-shaped emission distribution are too thin for models F0 and F1 (at 299, 454, $508 \mathrm{~km} \mathrm{~s}^{-1}$ ). The difference between models F2 and F3 is less obvious in the channel maps.

We conclude that models F2 and F3 reproduce the selected channel maps best.

\subsection{Atomic gas distribution}

The atomic gas distributions of the models with different flares F0 to F3 are shown in Fig. C.6 for the D and C+D array data. While the inner high column density HI disk is barely affected by the different flare models, the main differences are observed at the lowest column densities. Only models F2 and F3 show the observed extent perpendicular to the major axis. We note that the largest vertical extent occurs along the minor axis (owing to axisymmetry) in the models, whereas it occurs at an offset of $\sim 4^{\prime}$ along the major axis in our observations. This suggests that either the observed HI flare is not axisymmetric or the disk volume is not uniformly populated by atomic gas. As observed in all model maps, two gas blobs, which we interpret as the lobes of a ring structure, are located at the extremities of the gas disk $\left(R=11^{\prime}=24.6 \mathrm{kpc}\right)$. By construction, they are vertically offset from the disks major axis.

We thus conclude that models F2 and F3 show the best resemblance to our HI observations with a slight preference for model F3 (Sect. 5.1). In the following we refer to the model with the elliptical component, with an inclination varying by $10^{\circ}$, and flare F3 as the best-fit model (F3+E+INC).
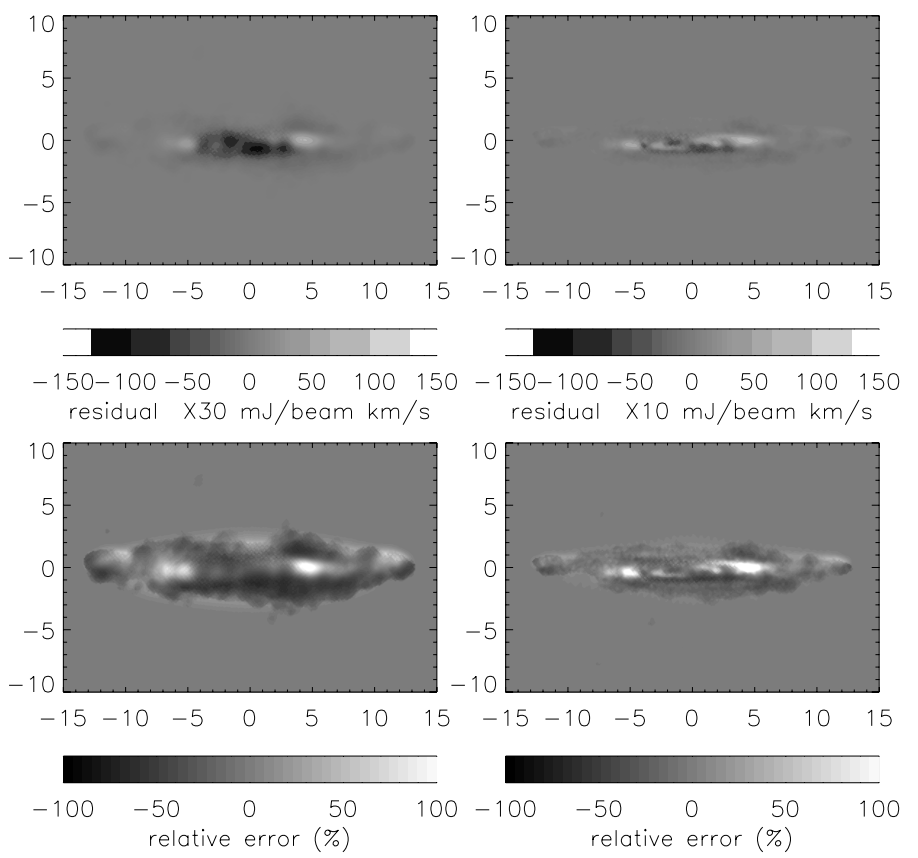

Fig. 10. Upper panels: residual column density from the best-fit model. Lower panels: relative residual column density from the best-fit model. Left: D array; right: $\mathrm{C}+\mathrm{D}$ array data. The offsets along the $x$ and $y$ axes are in arcmin.

The relative and absolute residual column density of the bestfit model are presented in Fig. 10 for the D and C+D data separately. Most residuals are asymmetric with respect to the major and minor axes and most probably caused by non-axisymmetric features in the observed atomic gas surface density. Within the inner $4^{\prime}$, negative residuals are located at positive/negative offsets on the $x / y$ axis and negative/positive offsets on the $x / y$ axis. These residuals are probably due to the spiral structure of the gas disk. For galactic radii between $4^{\prime}$ and $6^{\prime}$, the residuals are mostly positive.

\subsection{The rotation velocity lag}

Since the gas disk has a significant vertical extent only at galactic radii $>12 \mathrm{kpc}$, the effects of the lag are only visible in pv1-pv3 and pv7-9, shifting the emission distribution toward velocities closer to the systemic velocity. This only improves the resemblance between our model and observations for the outermost radius (pv1 and pv9). The addition of the rotation velocity lag changes the emission distribution significantly in four channels $\left(186,248,537\right.$, and $\left.599 \mathrm{~km} \mathrm{~s}^{-1}\right)$, but does not significantly improve the resemblance between our models and observations.

We conclude that a rotation velocity lag is not a necessary ingredient for our model.

\subsection{Atomic gas halo}

The origins of HI halos are thought to be (i) a continuous galactic fountain flow, where gas is pushed up by stellar activity, travels through the halo and eventually falls back to the disc (Fraternali \& Binney 2006) or (ii) external gas accretion. For case (i) we expect an HI halo within a radius of $R<10 \mathrm{kpc}$ where the molecular gas surface density and star formation activity is high (Figs. 5 and 13). For case (ii) the halo can extend to 
larger galactic radii. However, it can be most clearly identified in the inner disk $R<10 \mathrm{kpc}$, where warps and flares are rare.

Is there an extended atomic gas halo around the thin gas disk inside the optical radius? To answer this question, we introduced an additional thick gas disk of the following form (Oosterloo et al. 2007):

$\rho_{\text {halo }}=\chi \rho_{\text {disk }} \frac{\sinh \left(z / z_{0}\right)}{\cosh \left(z / z_{0}\right)^{2}}$

where $\rho_{\text {disk }}$ is the density of the gas disk, and vertical $z_{0}$ the scale height in kpc. We used the following combinations of $\left(\chi, z_{0}\right)$ : $\mathrm{H} 1=(0.3,0.75), \mathrm{H} 2=(0.2,1.0)$, and $\mathrm{H} 3=(0.1,1.25)$. This thick gas halo does not show up in the position velocity diagrams nor in the selected channel maps of the low resolution (D array) model. However, it is clearly visible in the position velocity diagrams and channel maps of the inner disk (Fig. C.7 pv3, $\mathrm{pv} 4, \mathrm{pv} 6$, and $\mathrm{pv} 7)$ in the high resolution $(\mathrm{C}+\mathrm{D}$ array) model in form of a low column density envelop around the thin disk. The comparison with our preferred model (right panels of Fig. C.3) shows that this additional envelop is not present in the observed position velocity diagrams. This statement is confirmed by the direct comparison between the channel maps of the models with and without an $\mathrm{HI}$ halo (Fig. C.5).

We conclude that NGC 2683 does not host an extended HI halo around the optical and thin gas disk with column densities in excess of $\sim 3 \times 10^{19} \mathrm{~cm}^{-2}$. The addition of a velocity lag does not change our conclusion.

\section{Discussion}

The main ingredients of our best-fit model of the gas disk are (i) a thin disk; (ii) an elliptical surface density distribution of the gas disk; (iii) a slight warp in inclination between $10 \mathrm{kpc} \leq$ $R \leq 20 \mathrm{kpc}$; (iv) an exponential flare that rises from $0.5 \mathrm{kpc}$ at $R=9 \mathrm{kpc}$ to $4 \mathrm{kpc}$ at $R=15 \mathrm{kpc}$, stays constant until $R=$ $22 \mathrm{kpc}$, and decreases its height for $R>22 \mathrm{kpc}$ (Fig. 9); and (v) a low surface density gas ring with a vertical offset of $1.3 \mathrm{kpc}$. Based on the comparison between the high resolution model and observations, we exclude the possibility that there is an extended atomic gas halo around the optical and thin gas disk (Fig. C.7).

\subsection{Uncertainties of the derived parameters}

The absence of an objective measure for the goodness of our model fits led to it not being possible to give precise uncertainties for the derived model parameters. Here we give estimates based on our experience with the comparison between our models and observations: the inclination angle of the thin disk is uncertain to $\Delta i \sim 3^{\circ}$. The uncertainty of the rotation velocity $\Delta v_{\text {rot }} \sim 10 \mathrm{~km} \mathrm{~s}^{-1}$ is mainly given by the spectral resolution. The lower limit of the $K^{\prime}$ band mass-to-light ratio is determined by the typical velocity dispersion of nearby spiral galaxies. We estimate the uncertainty to be $\Delta\left(M / L_{K^{\prime}}\right) \sim 0.3$. Thus, our preferred value of $M / L_{K^{\prime}}=0.9 \pm 0.3$ is significantly higher than the mean value of the DiskMass Survey $\left(M / L_{K^{\prime}}=0.31 \pm 0.07\right)$. Only one galaxy in this surveys has a comparable mass-to-light ratio. The uncertainty of the rotation curve of the dark matter halo is dominated by the uncertainty of the mass-to-light ratio in the inner disk $\left(\Delta v_{\mathrm{DM}} \sim 20-30 \mathrm{~km} \mathrm{~s}^{-1}\right)$. At $R>10 \mathrm{kpc}$, the latter uncertainty decreases and the uncertainty of the total rotation velocity is no longer negligible, yielding $\Delta v_{\mathrm{DM}} \sim 15 \mathrm{~km} \mathrm{~s}^{-1}$.

For the disk warp (inclination and position angle), we used a limited number of characteristic profiles. The best-fit profile of

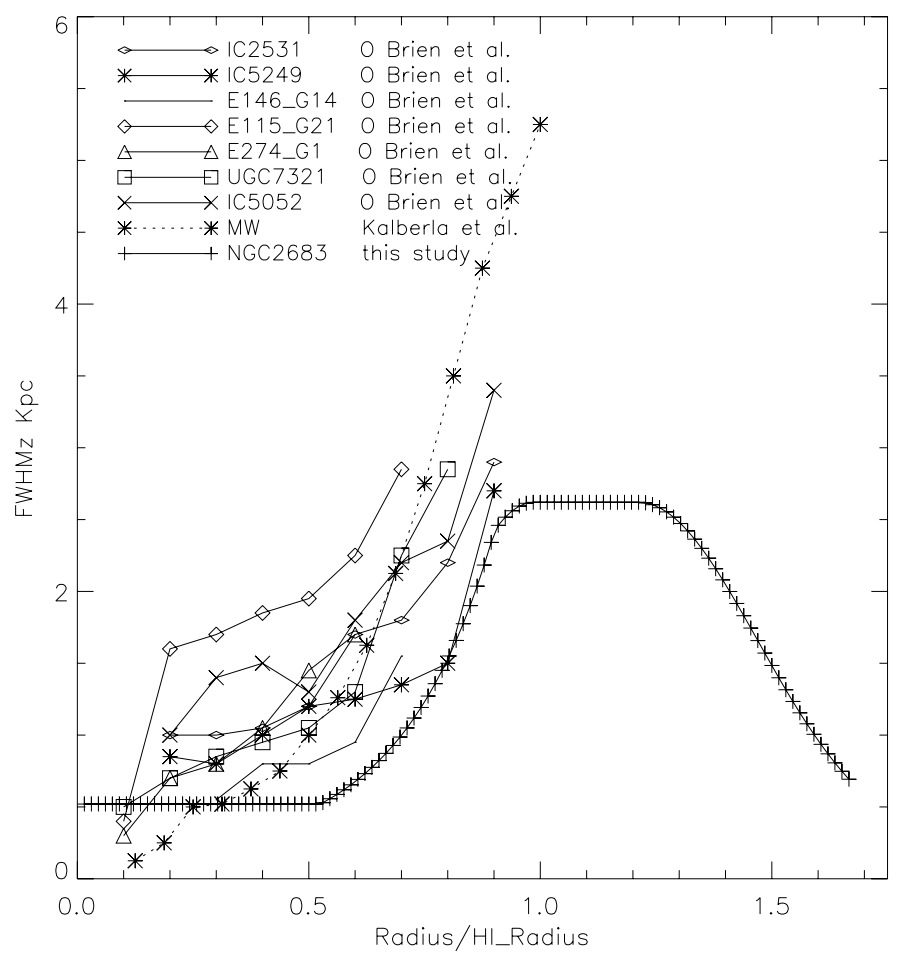

Fig. 11. Comparison between the flare of the gas disk and those of other spiral galaxies.

the inclination angle (Fig. 8) has a maximum variation of $10^{\circ}$. We estimate the uncertainty on the radial variation of the profile to be on the order of $30 \%$. We did not observe any improvement in the model fit by a variation in the position angle. The uncertainty on this profile is about $3-4^{\circ}$. For the disk flare, we also used a limited number (4) of characteristic profiles. Since the model with flare F3 (Fig. 9) reproduces our observations better (see Sect. 5.1), we suggest that the height of the flaring disk decreases at large galactic radii. We can give a lower limit for the slope of the flaring part (model F1 is discarded) and a tendency to prefer a flare that does not rise too steeply (model F3 is somewhat preferred to model F1). Thus a flare profile between model profiles F2 and F3 is possible. The maximum height of the flare is determined by the moment 0 maps and the $\mathrm{D}$ array position velocity diagrams. It is uncertain to a fraction of the $\mathrm{C}+\mathrm{D}$ array resolution, i.e. $\sim 0.3 \mathrm{kpc}$.

\subsection{Comparison with other $\mathrm{HI}$ flares}

In Fig. 11 we compare the flare of NGC 2683 with that of the Galaxy (Kalberla \& Kerp 2009) and other nearby spiral galaxies (O'Brien et al. 2010). For this comparison we set the HI radius of NGC 2683 to $17 \mathrm{kpc}$. The slope of NGC 2683's flare is comparable, but somewhat steeper than those of the other spiral galaxies. NGC 2683's maximum height of the flare is also comparable with those of the other galaxies. On the other hand, a saturation of the flare is only observed in NGC 2683.

\subsection{The vertical velocity dispersion of the gas}

We can go another step forward and calculate the velocity dispersion of the gas within the disk assuming that it is vertically sustained by turbulent motions. If vertical pressure equilibrium 

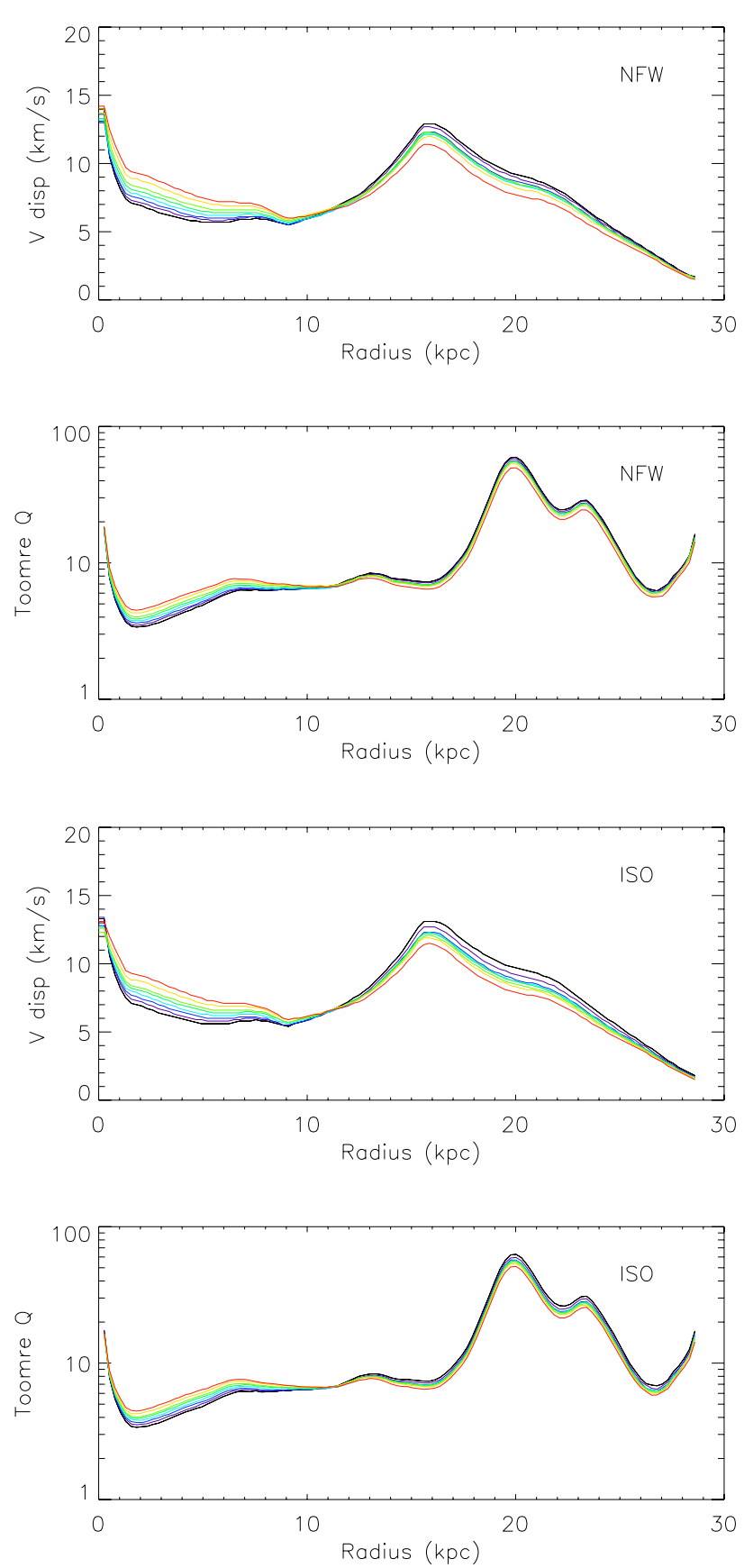

Fig. 12. Upper panel: vertical velocity dispersion of the gas halo of NGC 2683. Lower panel: derived Toomre $Q$ parameter. NFW halo and isothermal halo (ISO). The colors indicate different $K^{\prime}$ band mass-tolight ratios from blue (0.5) to red (1.3).

is assumed (see, e.g., Ostriker et al. 2010):

$\rho v_{\text {turb }}^{2}=\frac{1}{2} \pi G \Sigma\left(\Sigma\left(1+\frac{\rho_{\mathrm{DM}}}{\rho}\right)+\Sigma_{*} \frac{v_{\text {turb }}}{v_{\text {disp }}^{*}}\right)$,

where $v_{\text {turb }}$ is the vertical turbulent velocity of the gas, $G$ the gravitation constant, $\Sigma=\rho H$ the gas surface density, $\rho$ the gas density, $\rho_{\mathrm{DM}}$ the dark matter density, $\Sigma_{*}$ the stellar surface density, and $v_{\text {disp }}^{*}$ the stellar vertical dispersion velocity. The dark matter density is derived from the decomposition of the rotation curve (Fig. 4) and $\rho_{\mathrm{DM}}=\left(v_{\mathrm{DM}} / R\right)^{2} /(4 \pi G)$. As before, we used $K^{\prime}$ band mass-to-light ratios from 0.5 to 1.3 . The stellar velocity dispersion is calculated assuming a constant vertical scale height of the stellar disk (Eq. (B3) of Leroy et al. 2008), which is 1/7.3 times the radial scale length (Kregel et al. 2002). Since the uncertainty of this fraction $(1 /(7.3 \pm 2.2))$ resembles that of the $K^{\prime}$ band mass-to-light ratio, we estimate the uncertainty of the velocity dispersion to be $\Delta v_{\text {turb }} \sim 2 \mathrm{~km} \mathrm{~s}^{-1}$.

The derived vertical velocity dispersion of the gas decreases monotonically with increasing radius from $10 \mathrm{~km} \mathrm{~s}^{-1}$ in the inner disk $(R=1 \mathrm{kpc})$ to $7 \mathrm{~km} \mathrm{~s}^{-1}$ at $R=10 \mathrm{kpc}$ (Fig. 12). This is a typical behavior for nearby spiral galaxies (Tamburro et al. 2009; Fraternali et al. 2002). Within the flare ( $R=12 \mathrm{kpc}$ and $16 \mathrm{kpc})$, it increases to $12 \mathrm{~km} \mathrm{~s}^{-1}$ and then decreases monotonically. We believe that the increase is real, but its exact shape is mostly caused by our choice of the flare profile (Fig. 9), which leads to a satisfactory reproduction of our observations.

\subsection{The Toomre $Q$ parameter}

The Toomre stability parameter is

$Q=\frac{v_{\text {turb }} \Omega}{\pi G \Sigma_{\text {tot }}}$,

where $\Sigma_{\text {tot }}$ is the total gas surface density (see Sect. 4), and $\Omega=v_{\text {rot }} / R$. The Toomre parameter is about $Q=4-7$ for radii smaller than $10 \mathrm{kpc}$. It then increases slightly to $Q=8$ at $13 \mathrm{kpc}$, stays constant until $R=17 \mathrm{kpc}$, and then rises steeply to a maximum of $Q \sim 60$ at $20 \mathrm{kpc}$. The HI ring at the extremity of the gas disk shows a relatively low Toomre parameter of $Q \sim 6$. With such high $Q$ values, NGC 2683 is similar to NGC 3351 and NGC 2841 (Leroy et al. 2008).

\subsection{The missing $\mathrm{H} \mathrm{I}$ halo}

NGC 2683 does not host an HI halo around its optical disk and thin HI disk. This is consistent with the flocculent appearance of its disk on public HST images. Why does another equally massive spiral galaxy $\left(v_{\text {rot }} \sim 200 \mathrm{~km} \mathrm{~s}^{-1}\right.$ ), NGC 891, host an extended HI halo, whereas we can exclude the existence of such a gas halo for NGC 2683? The absence of a large-scale extended gaseous halo in the massive edge-on galaxy NGC 4565 (Heald et al. 2011) can be explained by its much lower energy injection rate compared to NGC 891 . Is this also the case for NGC 2683 ?

Within the optical disk $\left(R<9^{\prime}\right)$ of spiral galaxies, the thickness of gas disks can be explained well by energy injection via supernovae explosions (galactic fountains; Shapiro \& Field 1976; Bregman 1980) and the vertical restoring force. This force mainly depends on the disk mass surface density (gas and stars, see Eq. (9)). Because NGC 2683 has a lower stellar mass than NGC 891, we do not expect its disk restoring force to be higher than that of NGC 891. The energy injection rate is proportional to the local star formation rate $\Delta E /(\Delta t \Delta A) \propto \dot{\Sigma}_{*}$.

The star formation rate based on FIR emission of NGC 891 is $3.8 M_{\odot} \mathrm{yr}^{-1}$ (Popescu et al. 2004). NGC 2683's star formation rate based on radio continuum emission of $0.8 M_{\odot} \mathrm{yr}^{-1}$ (Irwin et al. 1999) is thus about five times lower than that of NGC 891. On the other hand, the flux ratios between NGC 891 and NGC 2683 in the IRAS $100 \mu \mathrm{m}$ band (Sanders et al. 2003) and Spitzer MIPS $70 \mu \mathrm{m}$ and $160 \mu \mathrm{m}$ bands are found to be around 5.5. Assuming distances of $9.5 \mathrm{Mpc}$ and $7.7 \mathrm{Mpc}$ for NGC 891 and NGC 2683, respectively, we infer the star formation of NGC 2683 to be about eight times lower than that of NGC 891.

To compare the local energy injection of the two galaxies, we show the Spitzer MIPS $70 \mu \mathrm{m}$ and $160 \mu \mathrm{m}$ surface brightness 


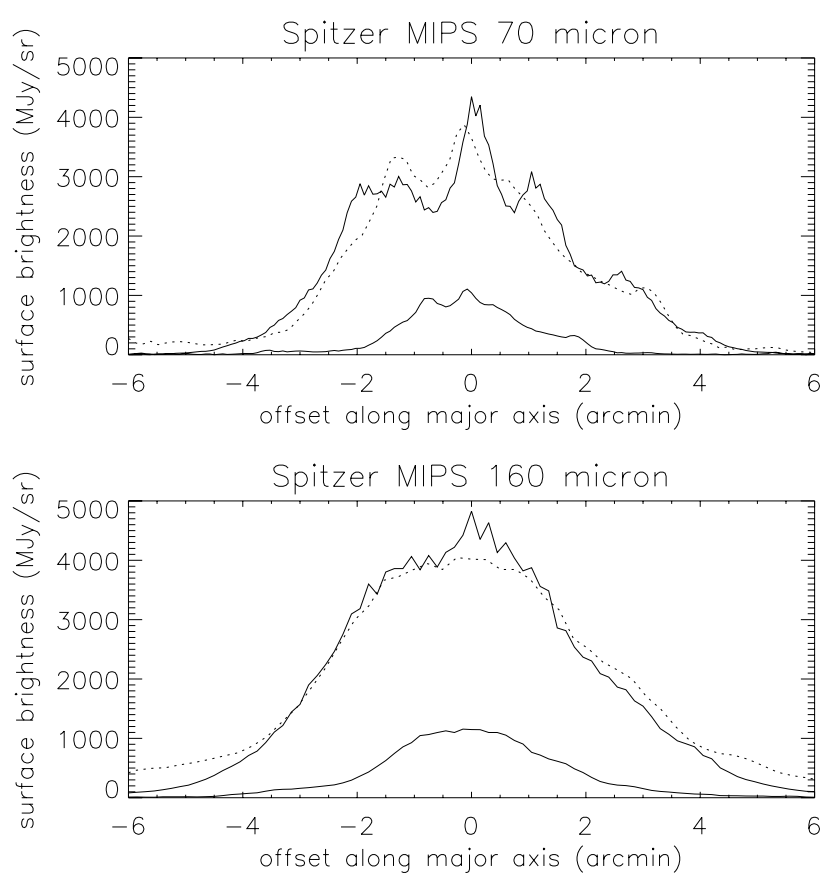

Fig. 13. Spitzer MIPS $70 \mu \mathrm{m}$ and $160 \mu \mathrm{m}$ surface brightness profiles of NGC 891 (upper solid lines) and NGC 2683 (lower solid lines) along the major axis summed over the minor axis. Dotted lines: profiles of NGC 2683 stretched by a factor 1.7 and multiplied by a factor 3.5 .

profiles along the galaxies' major axis summed over the minor axis in Fig. 13. NGC 2683 has a star forming disk that is about $1.7 \times \frac{9.5 \mathrm{Mpc}}{7.7 \mathrm{Mpc}}=2$ times less extended than NGC 891. This is expected based on the smaller scale length of the stellar disk (1.5 kpc compared to $4 \mathrm{kpc}$ for NGC 891; Schechtman-Rook \& Bershady 2013). The nearly 3.5 times lower surface brightness of the edge-on profile translates into a 3.5/2.0 = 1.8 times lower local star formation rate or energy injection rate of NGC 2683 than for NGC 891. In the innermost disk where the local star formation rate is high, the deep galactic gravitational potential probably prevents the formation of a significant Hi halo.

We conclude that the small extent of the star forming disk and the low local star formation rate of NGC 2683 explains the absence of an extended HI halo around the optical disk. Indeed, the HI halo of NGC 891 is the most prominent at galactic radii between 2.5 and $15 \mathrm{kpc}$ (Fig. 13 of Oosterloo et al. 2007), whereas the star forming disk of NGC 2983 only extends to $\sim 3 \mathrm{kpc}$.

\subsection{How to sustain the flaring HI disk}

NGC 2683's gas disk is flaring so is very thick at radii well beyond the optical radius where the rate of star formation is very low or even nonexistent. A galactic-fountain origin for the flare gas beyond the optical radius is thus excluded. For the majority of the flare region, the gas velocity dispersion is well above the speed of sound $\left(\sim 6 \mathrm{~km} \mathrm{~s}^{-1}\right)$. To maintain the gas velocity dispersion at this high level, four physical mechanisms can be suggested:

- energy injection by supernovae,

- magneto-rotational instabilities (Balbus \& Hawley 1991),

- ISM stirring by dark matter substructure,

- external gas accretion.
In the following we compare the energy injection rates of these mechanisms with the energy dissipation rate via turbulence.

Energy injection by supernovae: Following Vollmer \& Beckert (2003) energy flux conservation between the energy injection via supernovae and the energy dissipation rate via turbulence is

$\Sigma v \frac{v_{\text {turb }}^{2}}{l_{\text {driv }}^{2}}=\xi \dot{\Sigma}_{*}$,

where $v=v_{\text {turb }} l_{\text {driv }}$ is the gas viscosity, $l_{\text {driv }}$ the turbulent driving length scale, and $\xi=3.6 \times 10^{-8} \mathrm{pc}^{2} \mathrm{yr}^{-2}$ is normalized by the Galactic supernova rate. For a driving length scale of $l_{\text {driv }}=1 \mathrm{kpc}$, a turbulent velocity of $v_{\text {turb }}=8 \mathrm{~km} \mathrm{~s}^{-1}$, and a gas surface density of $\Sigma=0.5 M_{\odot} \mathrm{pc}^{-2}$, we obtain $\dot{\Sigma}_{*}=8 \times$ $10^{-12} M_{\odot} \mathrm{yr}^{-1} \mathrm{pc}^{-2}$. Such levels of star formation are observed in the outer disks of nearby spiral galaxies (Bigiel et al. 2010). The energy injection rate is $\Delta E_{*} /(\Delta A \Delta t) \sim 2 \times 10^{-8} \mathrm{erg} \mathrm{cm}^{-2} \mathrm{~s}^{-1}$.

Magneto-rotational instabilities: Following Tamburro et al. (2009), the energy injection rate by magneto-rotational instabilities for a disk height of $1 \mathrm{kpc}$, a magnetic field strength of $1 \mu \mathrm{G}$, and an angular velocity of $\Omega=10^{-8} \mathrm{yr}^{-1}$ are $\Delta E_{\mathrm{MRI}} /(\Delta A \Delta t) \sim$ $2 \times 10^{-8} \mathrm{erg} \mathrm{cm}^{-2} \mathrm{~s}^{-1}$. The energy injection due to magnetorotational instabilities is thus comparable to the one caused by supernovae.

ISM stirring by dark matter substructure: For the stirring of the gas disk by the dark matter substructure, we can estimate the energy deposited by a dark matter subhalo crossing the disk is $\Delta E \sim \epsilon M_{\text {halo }} v^{2}$. The timescale of interactions between a halo of mean mass $M_{\text {halo }}$ is $t_{\text {coll }}$. The energy injection rate is thus

$\frac{\Delta E}{\Delta A \Delta t}=\frac{N \epsilon M_{\text {halo }} v^{2}}{\pi R^{2} t_{\text {coll }}}$

We assume $v=150 \mathrm{~km} \mathrm{~s}^{-1}, M_{\text {halo }}=10^{7} M_{\odot}, R=20 \mathrm{kpc}$, and a collision timescale $t_{\text {coll }} \sim \pi R / v \sim 4 \times 10^{8}$ yr. With $N \sim 20$, a fraction of $\epsilon \sim 0.03$ of the halo's kinetic energy has to be injected into the ISM to balance the energy dissipation rate via turbulence.

External gas accretion: in the case of external accretion, one can estimate the necessary infall velocity $v_{\text {infall }}$ to maintain local gas turbulence via the following energy flux conservation equation:

$\Sigma \frac{v_{\text {turb }}^{3}}{H}=\frac{\dot{M}}{\Delta A} v_{\text {infall }}^{2}$,

where $\dot{M} \sim 0.2 M_{\odot} \mathrm{yr}^{-1}$ (Sancisi et al. 2008) is the external mass accretion rate, $\Sigma=0.5 M_{\odot} \mathrm{pc}^{-2}, H=3 \mathrm{kpc}$, and $\Delta A \sim$ $\pi(20 \mathrm{kpc})^{2}$. With these numbers we obtain $v_{\text {infall }} \sim 24 \mathrm{~km} \mathrm{~s}^{-1}$. The local dissipation timescale of turbulence is close to the crossing time $t_{\text {cross }} \sim H / v_{\text {turb }} \sim 3 \times 10^{8} \mathrm{yr}$ (Mac Low et al. 1999). The timescale of external accretion is $t_{\text {acc }} \sim M_{\text {outerHI }} / \dot{M} \sim 3 \mathrm{Gyr}$, where $M_{\text {outerHI }} \sim 6 \times 10^{8} M_{\odot}$ is the mass of the flaring part of the gas disk. Thus, it is only possible to maintain ISM turbulence by external gas accretion if the local dissipation timescale of turbulence is decreased by a factor of about ten with respect to its value for fully developed turbulence in a star forming disk.

For accreting thick gas tori around active galactic nuclei, Vollmer \& Davies (2013) suggest that massive and rapid gas accretion can lead to adiabatic compression of the ISM. This leads to (i) an increase in the gas velocity dispersion; (ii) intermittent turbulence; and (iii) quenching of star formation. Vollmer \& Davies (2013) propose that turbulent adiabatic compression can lead to intermittent turbulence and a subsequent decrease in the 
local dissipation timescale of turbulence (by the area filling factor of dense clumps or clouds). This mechanism might also act in the flaring gas disk of NGC 2683.

From these estimates none of the suggested mechanisms, which might be responsible for the driving of ISM turbulence, can be discarded. All effects might contribute to setting the overall velocity dispersion, but locally one of the four mechanisms might dominate according to the local conditions. The existence of the complex large-scale warping (Sect. 4) with asymmetries (Sect. 5.3) and small-scale structure (Sect. 3.1) might indicate that large scale external gas accretion has occurred and/or is occurring in NGC 2683. Alternatively, a triaxial dark matter halo can also induce a large-scale warp (e.g., Binney 1992). Since HI distributions of most spiral galaxies, which extend beyond the optical disk, show warps (Garcia-Ruiz et al. 2002) and are flared (O'Brien et al. 2010), we propose the following scenario for NGC 2683:

- Recent external gas accretion (within the last few Gyr) has added the atomic gas beyond the optical diameter (see van der Kruit 2007).

- Since the angular momentum of the infalling gas is not expected to be the same as that of the disk, a kinetic and spatial warp forms.

- During circularization, gas is compressed in colliding rotating gas streams/arms.

- If the compression is adiabatic, i.e. it increases the gas velocity dispersion, ISM turbulence becomes intermittent and star formation is quenched. In this case gas compression feeds and maintains the ISM turbulence.

- If the compressed turbulent gas can dissipate the injected mechanical energy, star formation proceeds in the compression region and the energy injection by subsequent $\mathrm{SN}$ explosions feeds and maintains ISM turbulence.

- Since we expect equipartition between the turbulent kinetic energy and the magnetic field energy, magneto-rotational instabilities might become important in regions where turbulence has enhanced the magnetic field.

This rather speculative scenario might be valid for other spiral galaxies with extended low surface density atomic gas. In addition, a triaxial halo might induce and/or modify a warp of the outer Hi disk.

\section{Conclusions}

New deep VLA D array HI observations of the nearby highly inclined spiral galaxy NGC 2683 have been presented. Archival C array data were processed and added to the new observations. In the D array data an rms noise level of $1.0 \mathrm{mJy} /$ beam is reached in a $5.16 \mathrm{~km} \mathrm{~s}^{-1}$ channel. The total HI mass of NGC 2683 is $M_{\mathrm{HI}}=1.42 \times 10^{9} M_{\odot}$. As already shown by Casertano \& van Gorkom (1991), the gas disk extends up to about three times the optical radius. At the extremity of the HI disk ( $27 \mathrm{kpc})$, we observe a ring-like structure whose projected surface density distribution has a blob-like structure. The position velocity diagram along the major axis does not show any counter-rotating gas.

To investigate the 3D structure of the atomic gas disk, we made different 3D models for which we produced model HI data cubes. These models have the following main components: (i) a thin gas disk with a thickness of 500 pc; (ii) different gas flares at galactic radii larger than $9 \mathrm{kpc}$ (Fig. 9); (iii) a possible warp of the disk; and (iii) an outer gas ring $(R>25 \mathrm{kpc})$.

The main ingredients of our best-fit model are: (i) a thin disk inclined by $80^{\circ}$, (ii) a crude approximation of spiral and/or bar structure by an elliptical surface density distribution of the gas disk; (iii) a slight warp in inclination between $10 \mathrm{kpc} \leq R \leq$ $20 \mathrm{kpc}$; (iv) an exponential flare that rises from $0.5 \mathrm{kpc}$ at $R=$ $9 \mathrm{kpc}$; to $4 \mathrm{kpc}$ at $R=15 \mathrm{kpc}$, stays constant until $R=22 \mathrm{kpc}$, and decreases its height for $R>22 \mathrm{kpc}$ (flare F3 in Fig. 9); and (v) a low surface density gas ring with a vertical offset of $1.3 \mathrm{kpc}$.

The slope of NGC 2683's flare is comparable, but somewhat steeper than those of other spiral galaxies. NGC 2683's maximum height of the flare is also comparable with those of other galaxies. On the other hand, a saturation of the flare is only observed in NGC 2683.

Based on the comparison between the high resolution model and observations, we excluded that there is an extended atomic gas halo around the optical and thin gas disk (Figs. C.3 and C.5). By comparing the disk properties (local star formation rate and mass surface density) of NGC 2683 with that of NGC 891, we concluded that the small extent of the star forming disk and the low local star formation rate of NGC 2683 might explain the absence of an extended Hi halo around the optical disk.

Under the assumption of vertical hydrostatic equilibrium, we derived the vertical velocity dispersion of the gas. As in other nearby galaxies (Tamburro et al. 2009; Fraternali et al. 2002), the velocity dispersion decreases monotonically with increasing radius within the optical disk. The velocity dispersion in the inner disk is about $8 \mathrm{~km} \mathrm{~s}^{-1}$.

Since the gas disk flares, i.e. is considerably thick, beyond the optical radius where star formation is low or even absent, it is improbable that galactic fountains are responsible for the high velocity dispersion (exceeding the thermal velocity dispersion). We estimated the energy dissipation rate due to turbulence and compared it to the energy injection rates due to (i) supernova explosions; (ii) magneto-rotational instabilities; (iii) ISM stirring by dark matter substructure; and (iv) external gas accretion. It is found that none of these mechanisms can be definitely discarded.

The existence of the complex large-scale warping (Sect. 4) and asymmetries (Sect. 5.3) indicates that large scale external gas accretion has occurred and/or is occurring in NGC 2683. Since most galaxies with HI distributions extending beyond the optical disk show warps (Garcia-Ruiz et al. 2002) and are flared (O'Brien et al. 2010), we propose a scenario where recent external gas accretion (within the last view Gyr) has added the atomic gas beyond the optical radius, in which a kinetic and spatial warp forms. During circularization, gas is compressed in colliding rotating gas streams/arms. If the compression is adiabatic, i.e. it increases the gas velocity dispersion, ISM turbulence becomes intermittent and star formation is expected to be quenched. In this case gas compression feeds and maintains the ISM turbulence. If the compressed turbulent gas can dissipate the injected mechanical energy, star formation proceeds in the compression region, and the energy injection by subsequent SN explosions feeds and maintains ISM turbulence.

Acknowledgements. We would like to thank the anonymous referee for constructive comments that helped to improve this article significantly.

\section{References}

Balbus, S. A., \& Hawley, J. F. 1991, ApJ, 376, 214

Barbieri, C. V., Fraternali, F., Oosterloo, T., et al. 2005, A\&A, 439, 947

Bell, E. F., McIntosh, D. H., et al. 2003, ApJS, 149, 289

Bigiel, F., Leroy, A., Walter, F., et al. 2010, AJ, 140, 1194

Binney, J. 1992, ARA\&A, 30, 51

Boomsma, R., Oosterloo, T. A., Fraternali, F., van der Hulst, J. M., \& Sancisi, R. 2005, A\&A, 431, 65

Boomsma, R., Oosterloo, T. A., Fraternali, F., van der Hulst J. M., \& Sancisi, R. 2008, A\&A, 490, 555 
Bournaud, F., Combes, F., Jog, C. J., \& Puerari, I. 2005, A\&A, 438, 507 Bregman, J. M. 1980, ApJ, 236, 577

Brinks, E., \& Burton, W. B. 1984, A\&A, 141, 195

Casertano, S., \& van Gorkom, J. H. 1991, AJ, 101, 1231

Chaves, T. A., \& Irwin, J. A. 2001, ApJ, 557, 646

Courteau, S., Dutton, A. A., van den Bosch, F. C., et al. 2007, ApJ, 671, 203

Dahlem, M., Lisenfeld, U., \& Rossa, J. 2006, A\&A, 457, 121

de Vaucouleurs, G., de Vaucouleurs, A., \& Corwin, J. R. 1976, Second reference catalogue of bright galaxies (Austin: University of Texas Press)

Elfhag, T., Booth, R. S., Höglund, B., et al. 1996, A\&AS, 115, 439

Fraternali, F., \& Binney, J. J. 2006, MNRAS, 366, 449

Fraternali, F., van Moorsel, G., Sancisi, R., \& Oosterloo, T. 2002, ApJ, 123, 3124

Garcìna-Ruiz, I., Sancisi, R., \& Kuijken, K. 2002, A\&A, 394, 769

Gentile, G., Jozsa, G. I. G., Serra, P., et al. 2013, A\&A, 554, A125

Heald, G., Józsa, Serra, P., et al. 2011, A\&A, 528, A118

Irwin, J. A., English, J., \& Sorathia, B. 1999, AJ, 117, 2102

Kalberla, P. M. W., \& Kerp, J. 2009, ARA\&A, 47, 27

Kamphuis, P., Rand, R. J., Józsa G. I. G., et al. 2013, MNRAS, 434, 2069

Kent, S. M. 1985, ApJS, 59, 115

Kregel, M., \& van der Kruit, P. C. 2004, MNRAS, 352, 787

Kregel, M., van der Kruit, P. C., \& de Grijs, R. 2002, MNRAS, 334, 646

Kuzio de Naray, R., Zagursky, M. J., \& McGaugh, S. S. 2009, AJ, 138, 1082

Leroy, A. K., Walter, F., Brinks, E., et al. 2008, AJ, 136, 2782

Lucy L. B. 1974, AJ, 79, 745

MacLow, M.-M. 1999, ApJ, 524, 169

Martinsson, T. P. K., Verheijen, M. A. W., Westfall, K. B., et al. 2013, A\&A, 557, A131

Matthews, L. D., \& Wood, Kenneth 2003, ApJ, 593, 721

Napier, P. J., Thompson, A. R., \& Ekers, R. D. 1983, IEEEP, 71, 1295

Nilson, P., 1973, Uppsala General Catalogue of Galaxies, Acta Universitatis Upsalienis, Nova Regiae Societatis Upsaliensis, Series v: a Vol.
O’Brien, J. C., Freeman, K. C., \& van der Kruit, P. C. 2010, A\&A, 515, A62

Oosterloo, T., Fraternali, F., \& Sancisi, R. 2007, AJ, 134, 1019

Ostriker, E. C., McKee, C. F., \& Leroy, A. K. 2010, ApJ, 721, 975

Peng, C. Y., Ho, L. C., Impey, C. D., \& Rix, H.-W. 2002, AJ, 124, 266

Pérez, I., Fux, R., \& Freeman, K. 2004, A\&A, 424, 799

Popescu, C. C., Tuffs, R. J., Kylafis, N. D., \& Madore, B. F. 2004, A\&A, 414, 45

Rand, R. J., \& Benjamin, R. A. 2008, ApJ, 676, 991

Rossa, J., \& Dettmar, R.-J. 2003, A\&A, 406, 493

Sancisi, R., Fraternali, F., Oosterloo, T., \& van der Hulst, T. 2008, A\&ARv, 15 189

Sanders, D. B., Mazzarella, J. M., Kim, D.-C., Surace, J. A., \& Soifer, B. T. 2003, AJ, 126, 1607

Schechtman-Rook, A., \& Bershady, M. A. 2013, ApJ, 773, 45

Shapiro, P. R., \& Field, G. B. 1976, ApJ, 205, 762

Skrutskie M. F., Cutri R. M., Stiening R., et al. 2006, AJ, 131, 1163

Sofue, Y., \& Rubin, V. 2001, ARA\&A, 39, 137

Springob, C. M., Haynes, M. P., Giovanelli, R., \& Kent, B. R. 2005, ApJS, 160, 149

Tamburro, D., Rix, H.-W., Leroy, A. K., et al. 2009, AJ, 137, 4424

Tonry, J. L., Dressler, A., Blakeslee, J. P., et al. 2001, ApJ, 546, 681

van der Hulst, \& J. M., Sancisi, R. 2005, Extra-Planar Gas, eds. R. Braun. (San Francisco), ASP Conf. Proc., 331, 139

van der Kruit, P. C. 2007, A\&A, 466, 883

Vollmer, B., \& Beckert, T. 2003, A\&A, 404, 21

Vollmer, B., \& Davies, R. I. 2013, A\&A, 556, A31

Walter, F., Brinks, E., de Blok, W. J. G., et al. 2008, AJ, 136, 2563

Warmels, R. H. 1988, A\&AS, 72, 427

Yamasaki, N. Y., Sato, K., Mitsuishi, I., \& Ohashi, T. 2009, PASJ, 61, 291

Young, J. S., \& Scoville, N. Z. 1991, ARA\&A, 29, 581

Zschaechner, L. K., Rand, R. J., Heald, G., Gentile, G., \& Jozsa, G. 2012, ApJ, 760,37 


\section{Appendix A: HI position velocity diagrams}

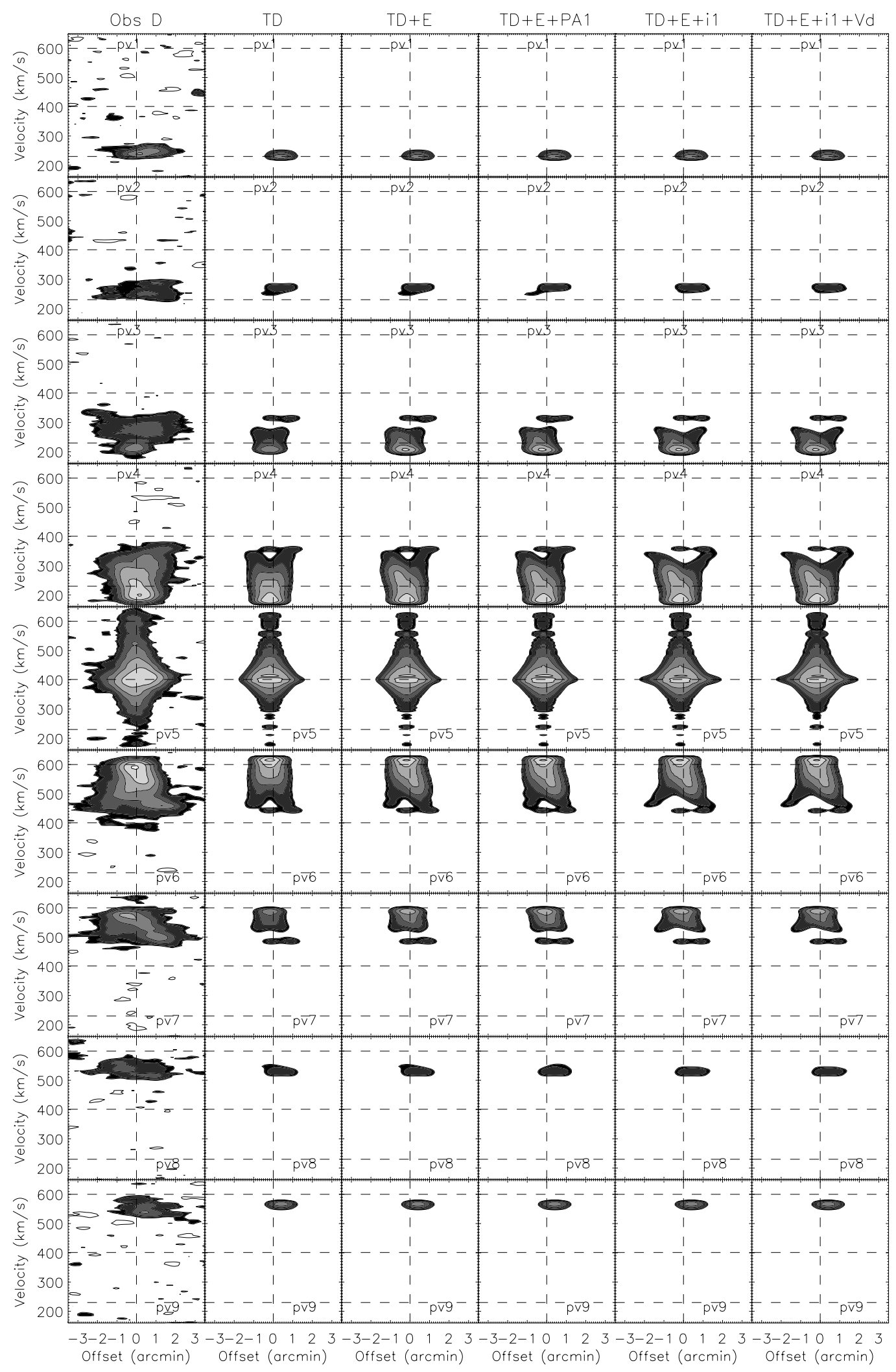

Fig. A.1. NGC 2683 HI D array and model position velocity diagrams. The model ingredients are a thin disk (TD), an elliptical component (E), a warp in position angle (PA), a warp in inclination (i), and a radially decreasing velocity dispersion (Vd). The contour levels are $(-2,2,3,6,12,24,48,96) \times 0.7 \mathrm{mJy} /$ beam. The resolution is $61^{\prime \prime} \times 51^{\prime \prime}$. 
B. Vollmer et al.: The flaring HI disk of NGC 2683

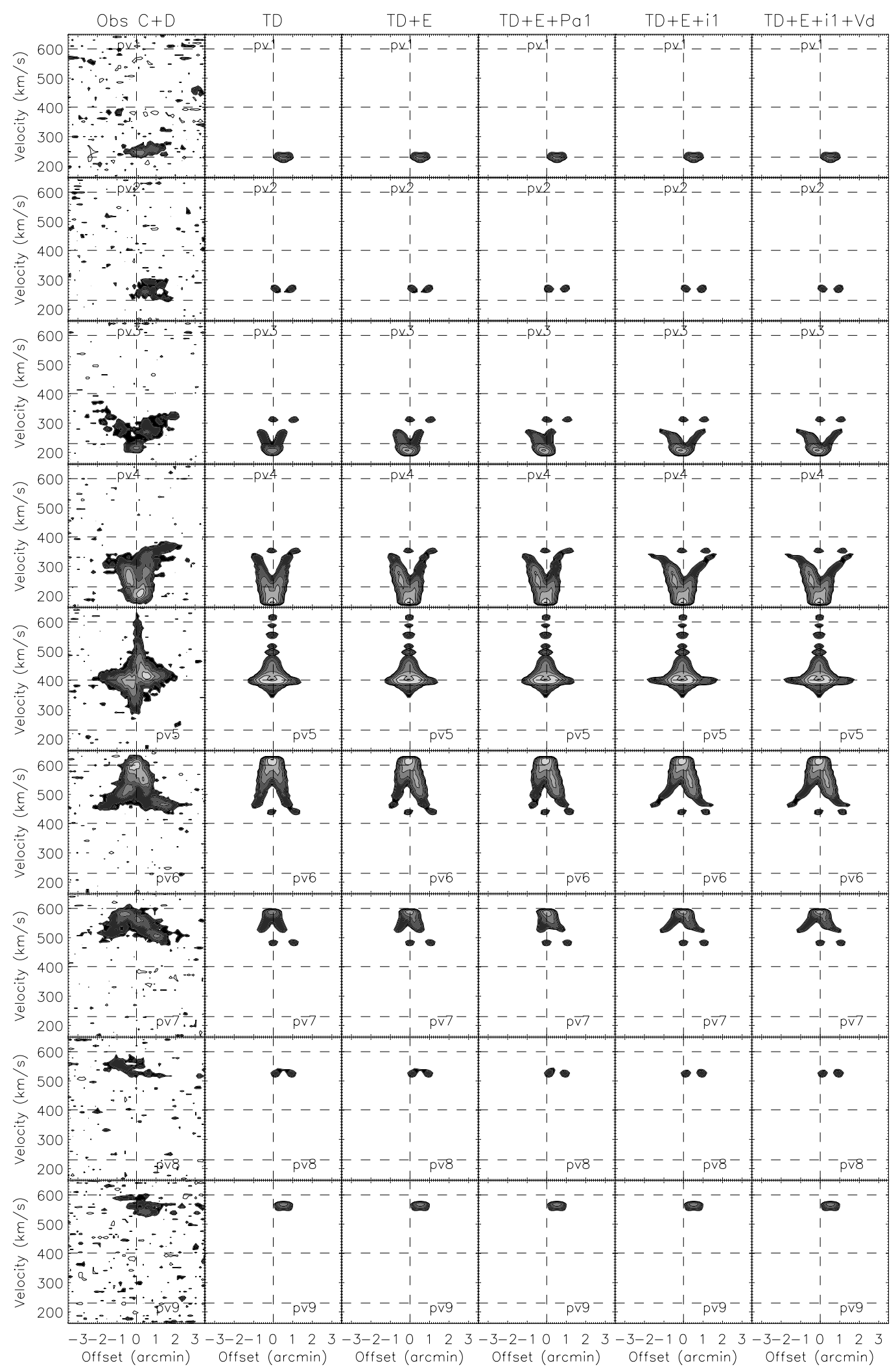

Fig. A.2. NGC 2683 HI C+D array and model position velocity diagrams. The model ingredients are a thin disk (TD), an elliptical component (E), a warp in position angle (PA), a warp in inclination (i), and a radially decreasing velocity dispersion (Vd). The contour levels are $(-2,2,3,6,12,24,48,96) \times 0.3 \mathrm{mJy} /$ beam. The resolution is $19^{\prime \prime} \times 18^{\prime \prime}$. 


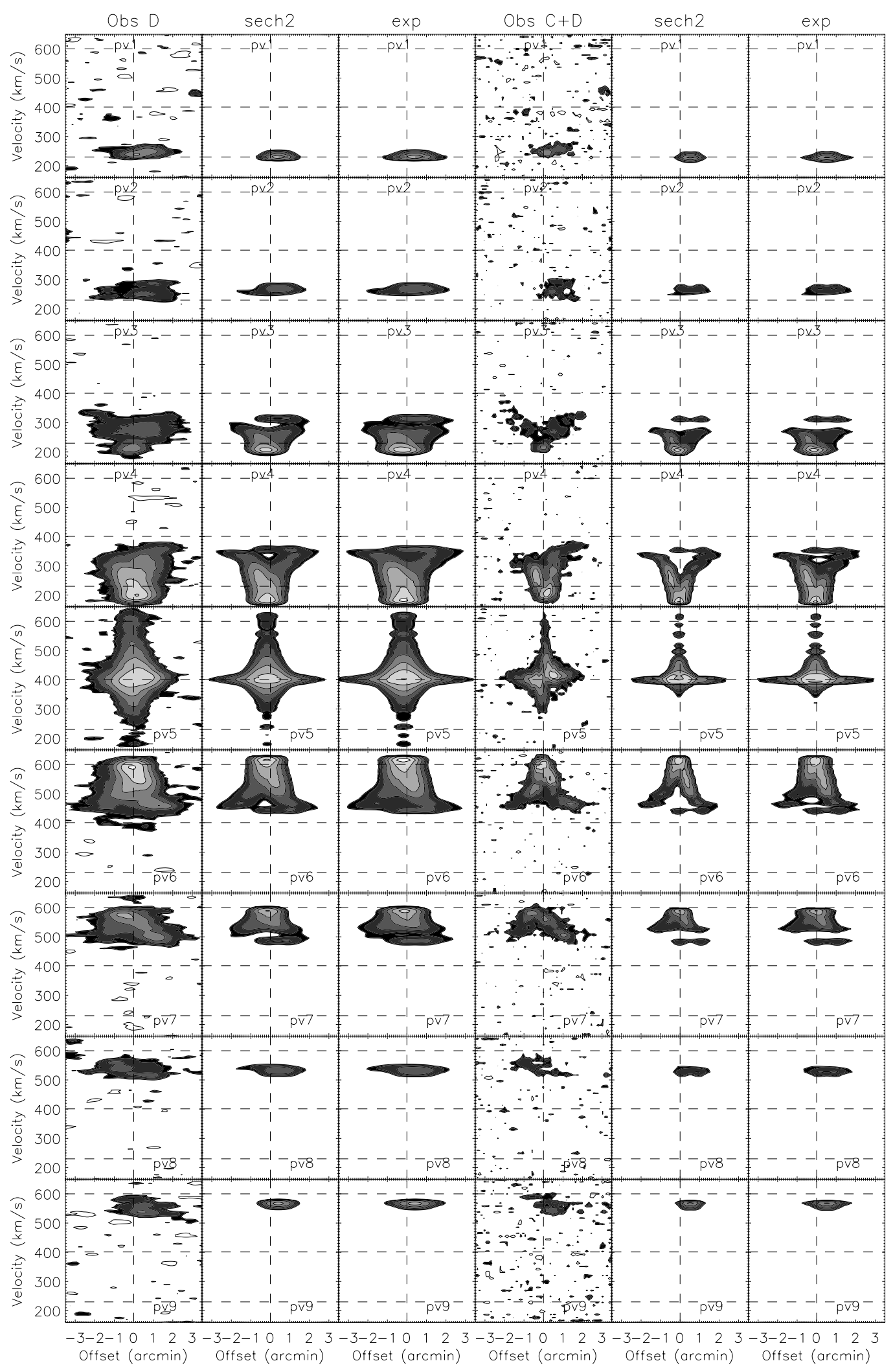

Fig. A.3. Models with different vertical structures of the gas disk ( $\mathrm{sech}^{2}$ and exponential). Left panels: NGC 2683 HI D array and model position velocity diagrams. The contour levels are $(-2,2,3,6,12,24,48,96) \times 0.7 \mathrm{mJy} /$ beam. The resolution is $61^{\prime \prime} \times 51^{\prime \prime}$. Models include a flare with $\mathrm{sech}^{2}$ and exponential vertical profiles. Right panels: NGC $2683 \mathrm{HI}$ C+D array and model position velocity diagrams. The contour levels are $(-2,2,3,6,12,24,48,96) \times 0.3 \mathrm{mJy} /$ beam. The resolution is $19^{\prime \prime} \times 18^{\prime \prime}$. Models include a flare with sech ${ }^{2}$ and exponential vertical profiles. 


\section{Appendix B: HI channel maps}

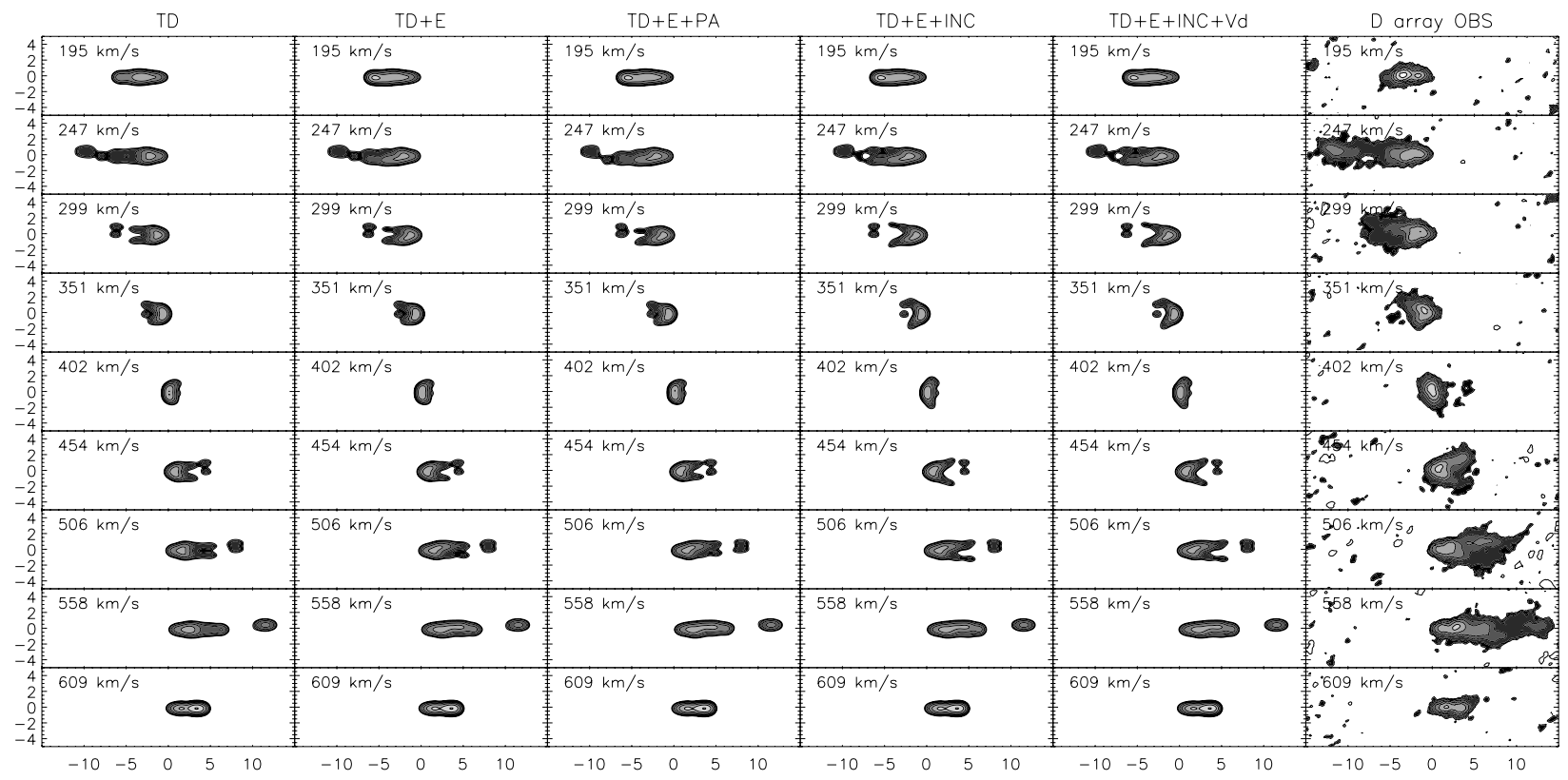

Fig. B.1. Selected HI D array and model channel maps. The model ingredients are a thin disk (TD), an elliptical component (E), a warp in position angle (PA), a warp in inclination (INC), and a radially decreasing velocity dispersion (Vd). The offsets along the $x$ and $y$ axes are in arcmin. The contour levels are $(-2,2,3,6,12,24,48,96) \times 0.8 \mathrm{mJy} / \mathrm{beam}$. The resolution is $61^{\prime \prime} \times 51^{\prime \prime}$. The galaxy was rotated by $45^{\circ}$.

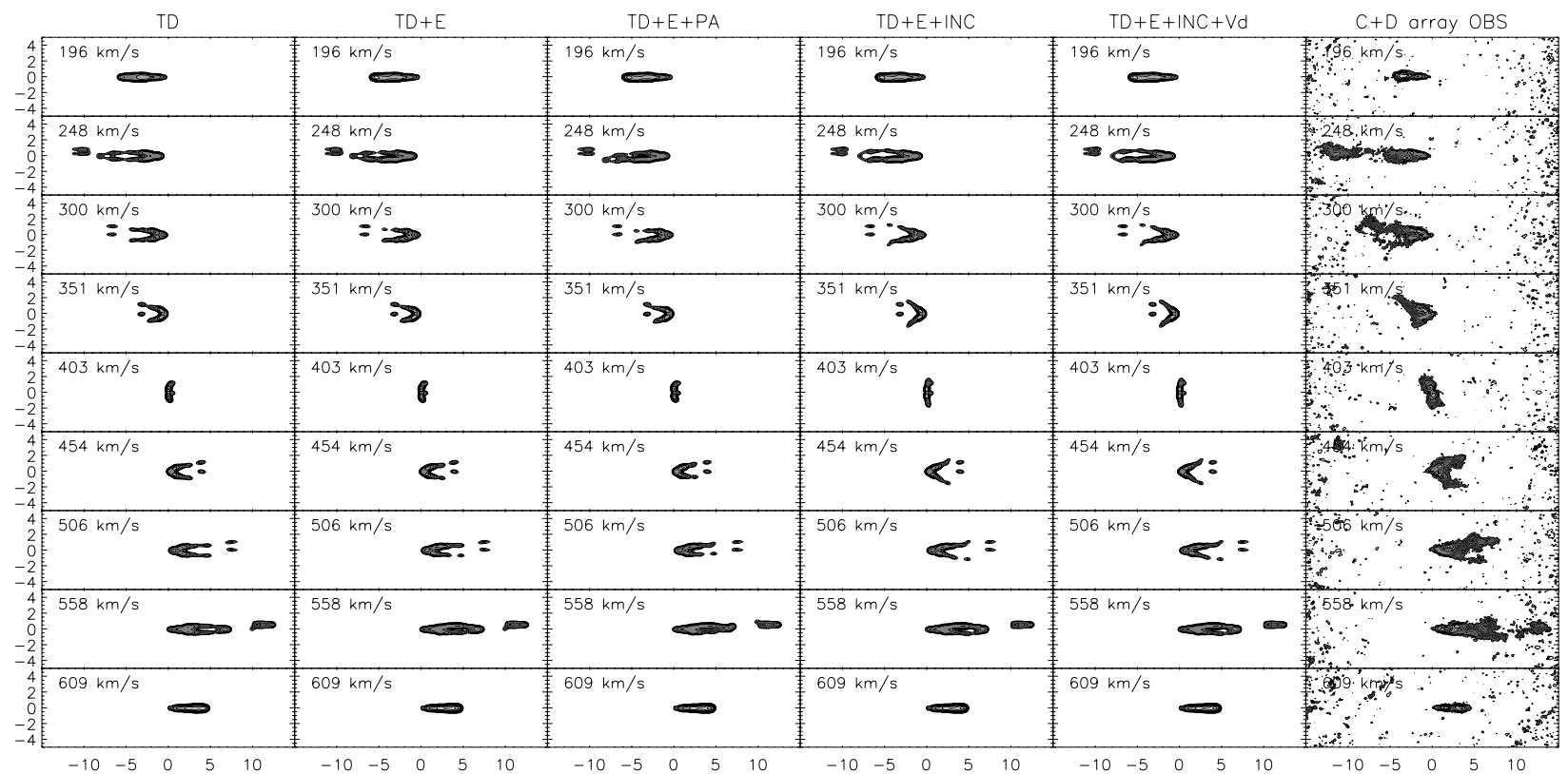

Fig. B.2. Selected HI C+D array and model channel maps. The model ingredients are a thin disk (TD), an elliptical component (E), a warp in position angle (PA), a warp in inclination (INC), and a radially decreasing velocity dispersion (Vd). The offsets along the $x$ and $y$ axes are in arcmin. The contour levels are $(-2,2,3,6,12,24,48,96) \times 0.4 \mathrm{mJy} /$ beam. The resolution is $19^{\prime \prime} \times 18^{\prime \prime}$. The galaxy was rotated by $45^{\circ}$. 


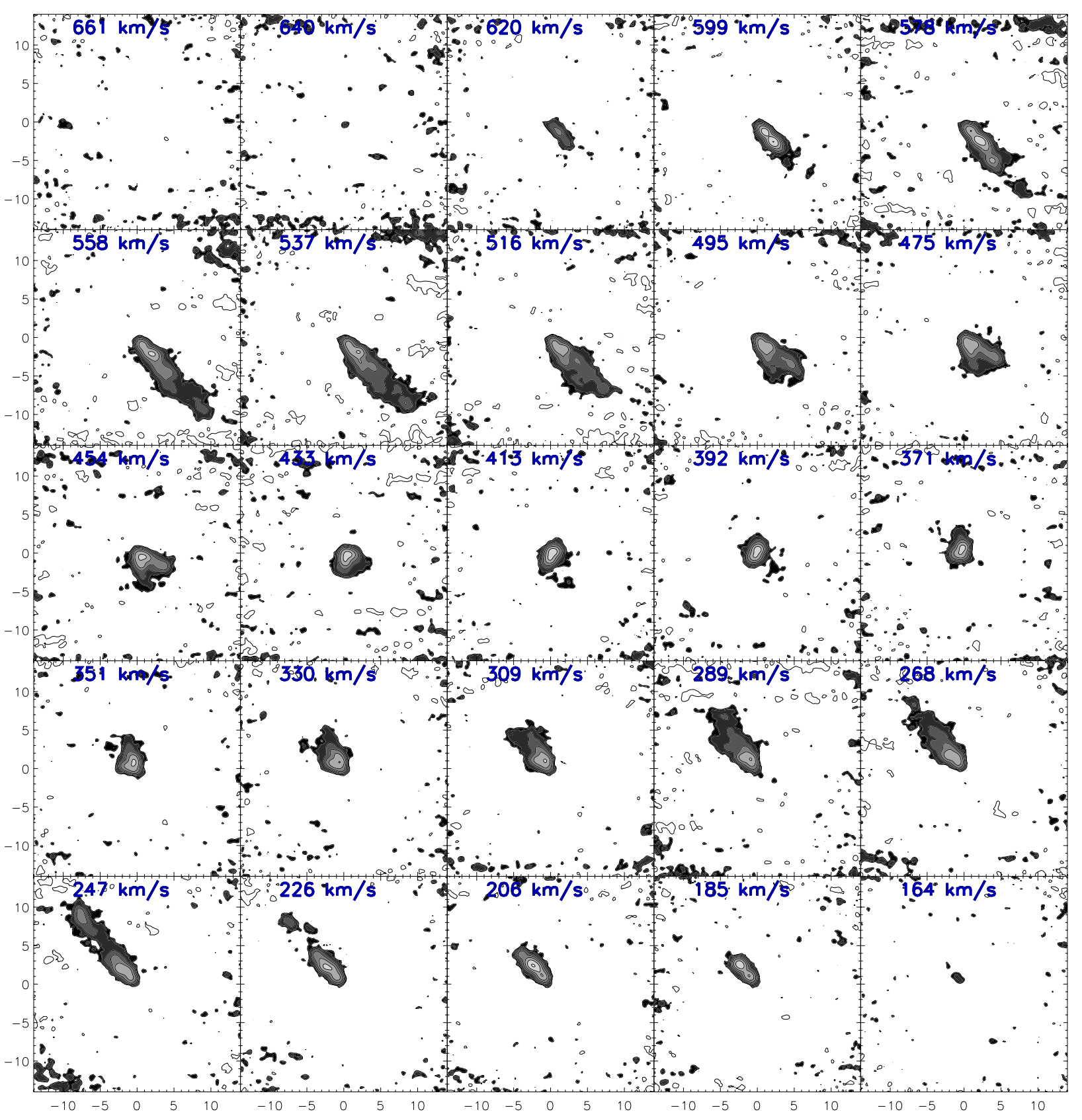

Fig. B.3. NGC $2683 \mathrm{HI}$ D array channels maps. The contour levels are $(-2,2,3,6,12,24,48,96) \times 0.8 \mathrm{mJy} /$ beam. The resolution is $61^{\prime \prime} \times 51^{\prime \prime}$. The offsets along the $x$ and $y$ axes are in arcmin. 


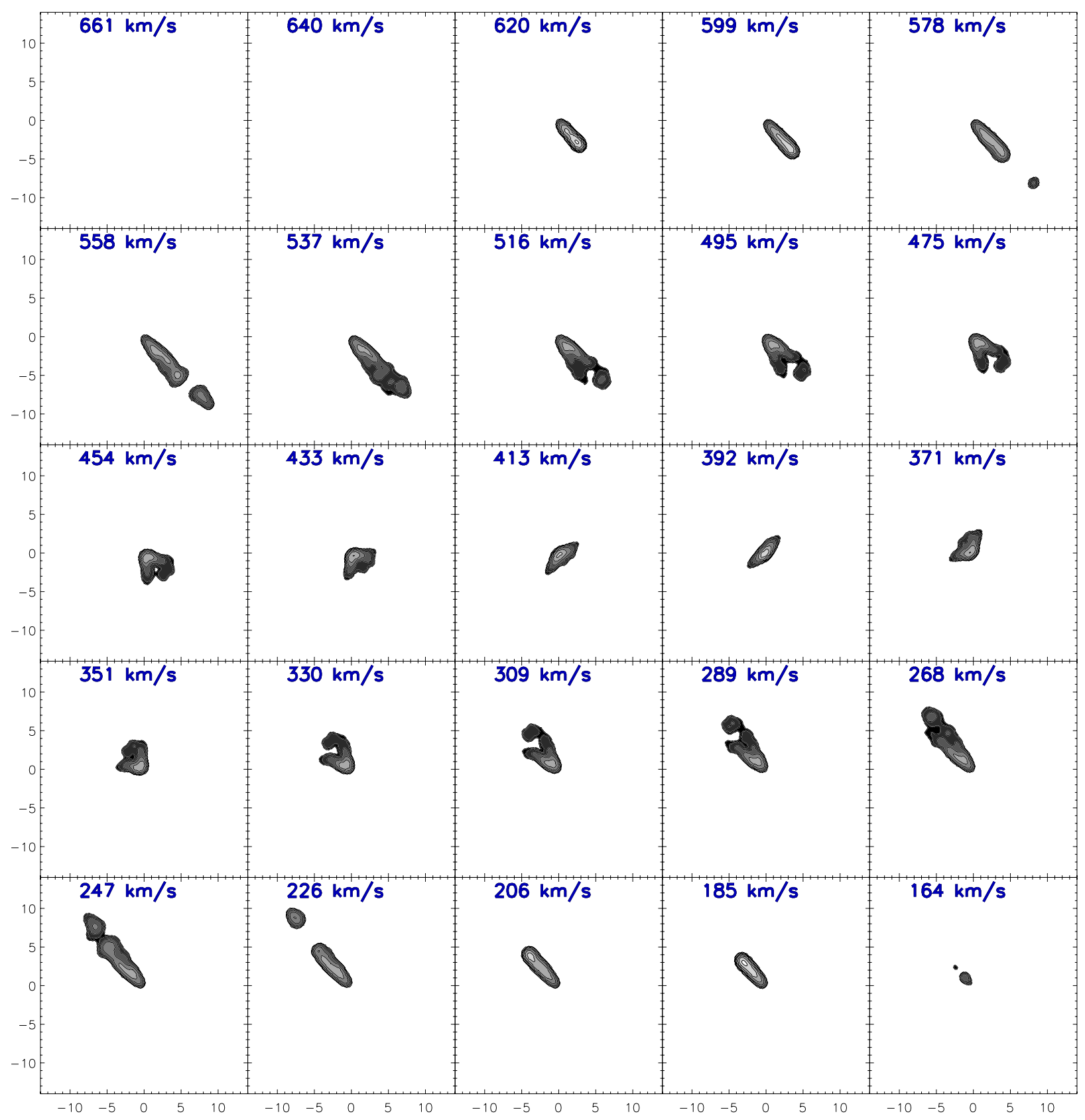

Fig. B.4. Best-fit model channel maps. The model ingredients are a thin disk (TD), an elliptical component (E), a warp in inclination (INC), a radially decreasing velocity dispersion $(\mathrm{Vd})$, and a flare $(\mathrm{F} 3)$. The resolution is $61^{\prime \prime} \times 51^{\prime \prime}$. The contour levels are $(-2,2,3,6,12,24,48,96) \times$ $0.8 \mathrm{mJy} /$ beam. The channels of the original data cube were averaged to yield a channel separation of $20 \mathrm{~km} \mathrm{~s}^{-1}$. The offsets along the $x$ and $y$ axes are in arcmin. 


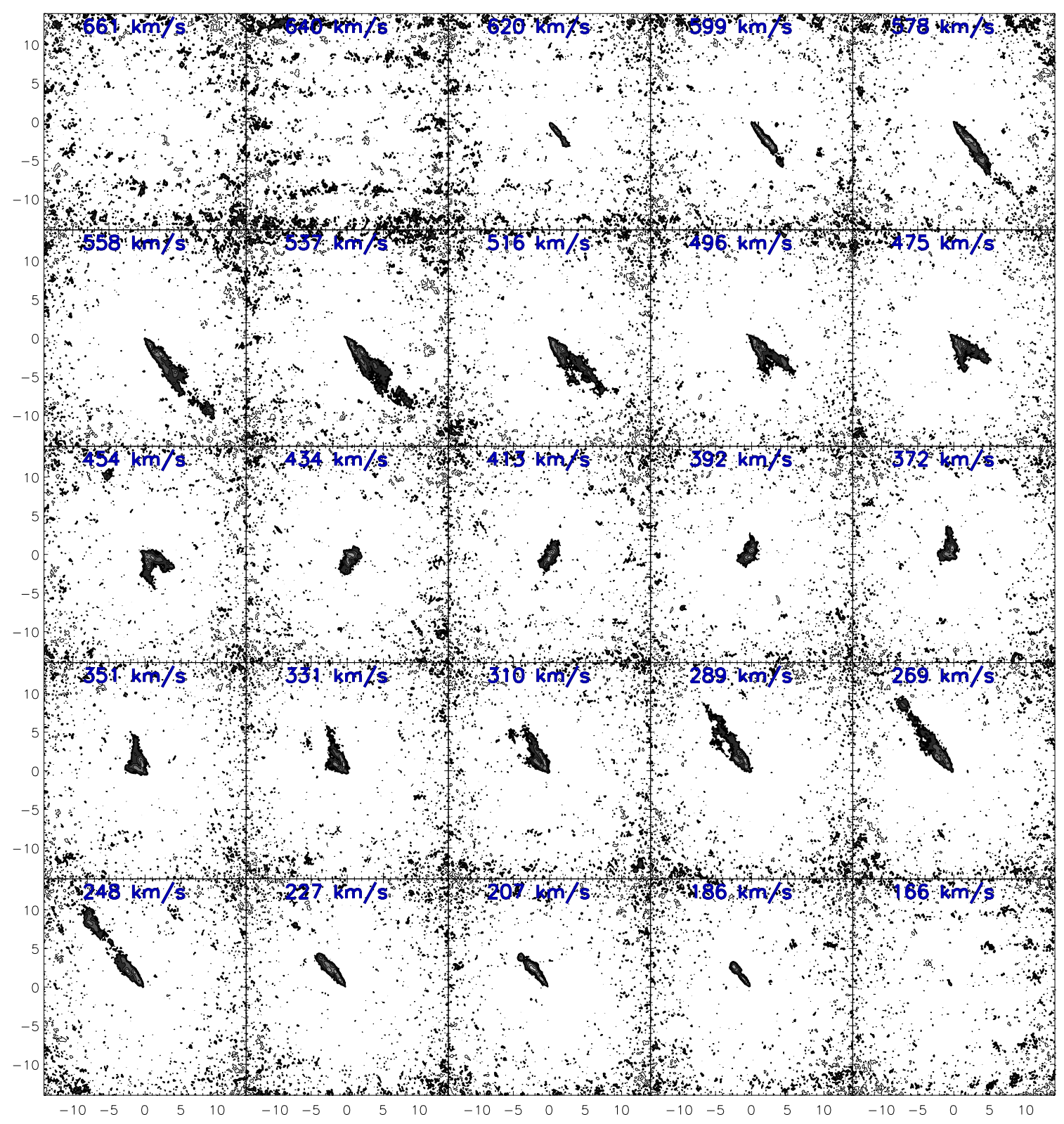

Fig. B.5. NGC $2683 \mathrm{HI}$ C+D array channels maps. The contour levels are $(-2,2,3,6,12,24,48,96) \times 0.4 \mathrm{mJy} / \mathrm{beam}$. The resolution is $19^{\prime \prime} \times 18^{\prime \prime}$. The offsets along the $x$ and $y$ axes are in arcmin. 


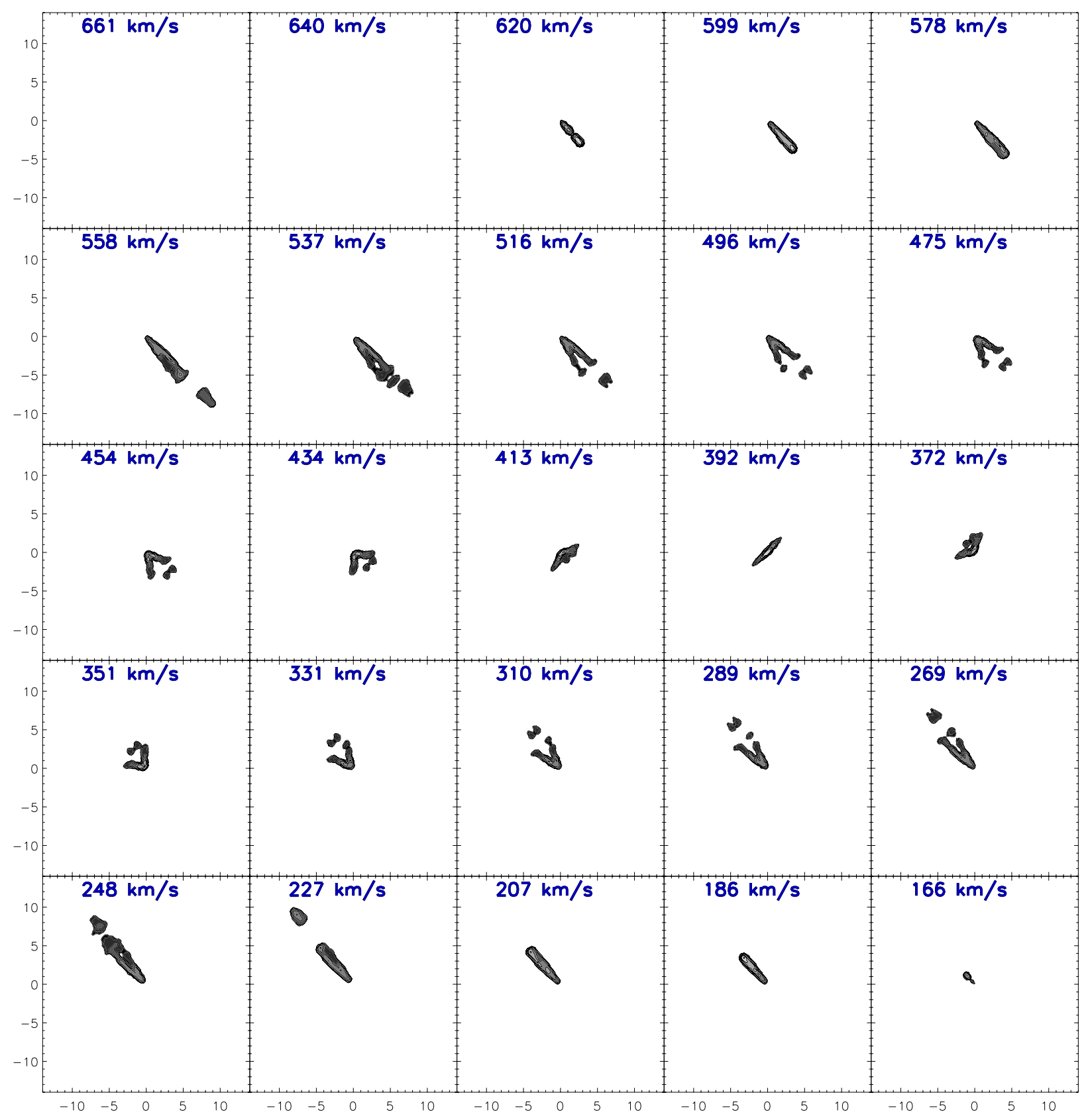

Fig. B.6. Best-fit model channel maps. The model ingredients are a thin disk (TD), an elliptical component (E), a warp in inclination (INC), a radially decreasing velocity dispersion $(\mathrm{Vd})$, and a flare $(\mathrm{F} 3)$. The contour levels are $(-2,2,3,6,12,24,48,96) \times 0.4 \mathrm{mJy} / \mathrm{beam}$. The resolution is $19^{\prime \prime} \times 18^{\prime \prime}$. The offsets along the $x$ and $y$ axes are in arcmin. 


\section{Appendix C: Additional figures}

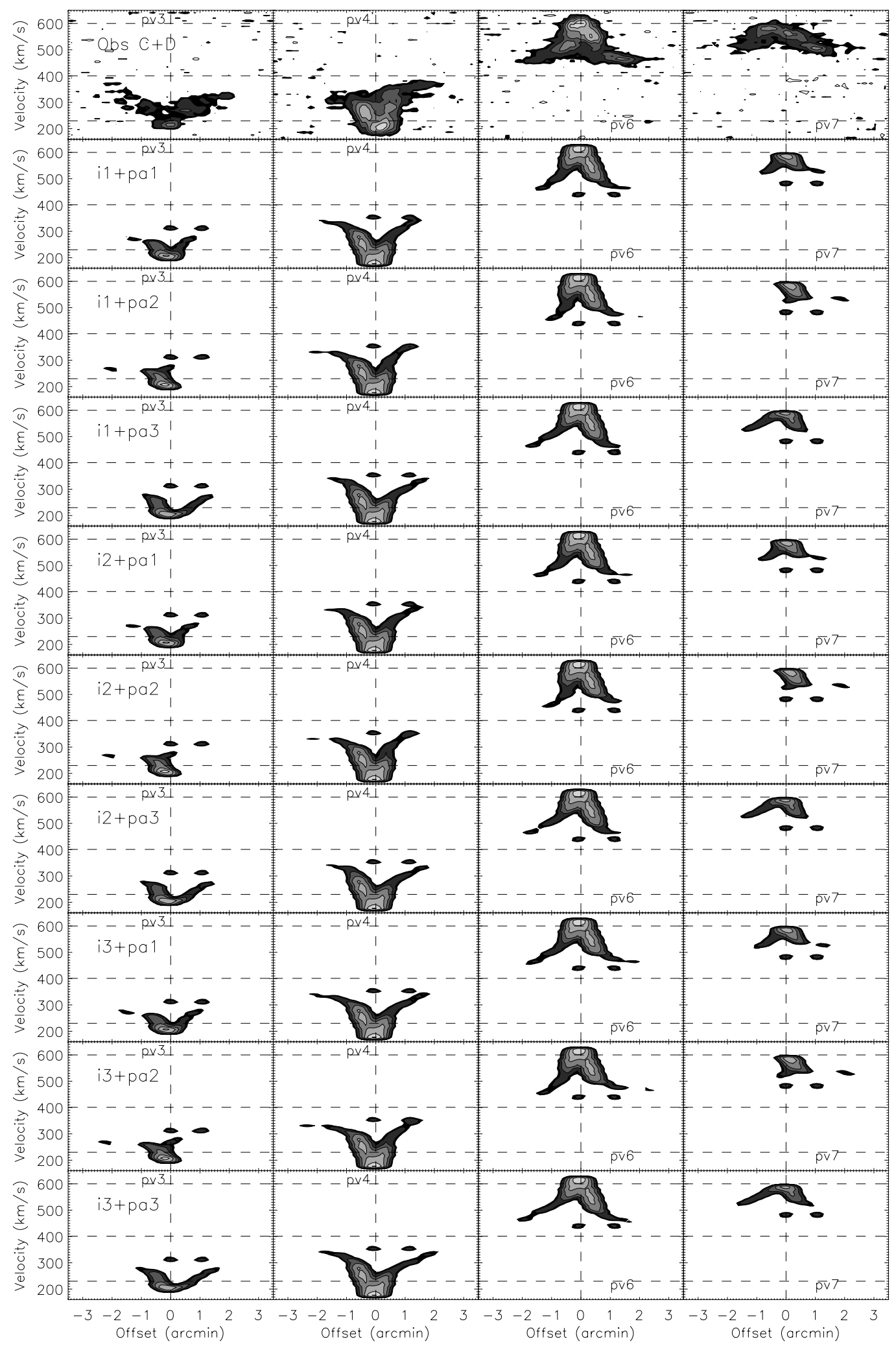

Fig. C.1. NGC $2683 \mathrm{HI}$ C+D array and model position velocity diagrams. The contour levels are $(-2,2,3,6,12,24,48,96) \times 0.3 \mathrm{mJy} / \mathrm{beam}$. The resolution is $19^{\prime \prime} \times 18^{\prime \prime}$. The different warp models refer to the profiles of Fig. 8 . 


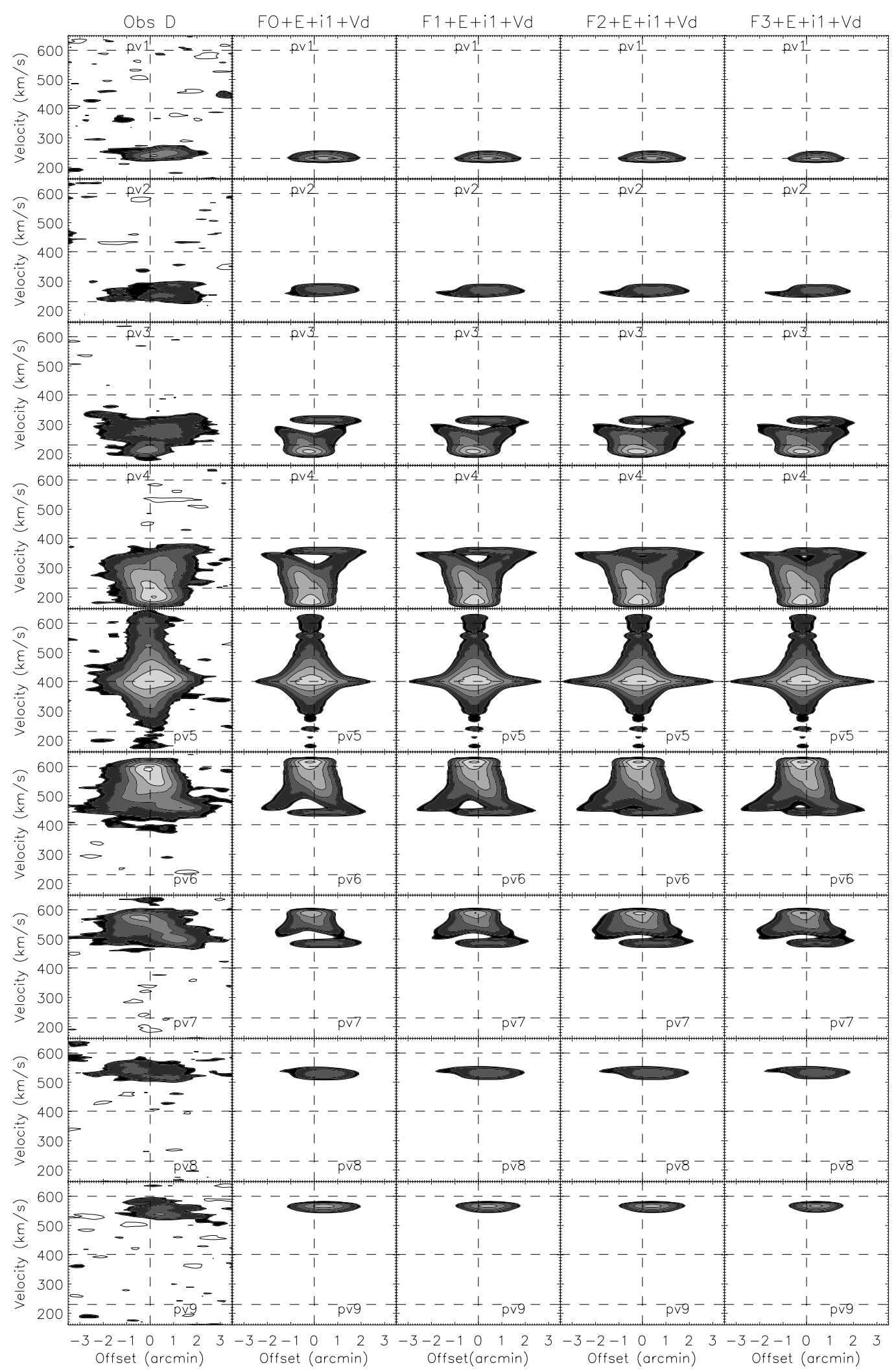

Fig. C.2. NGC 2683 HI D array and model position velocity diagrams. The model includes a thin disk, an elliptical component (E), a warp in inclination (i1), a radially decreasing velocity dispersion (Vd), and a flare (F0-F3; see Fig. 9). The contour levels are $(-2,2,3,6,12,24,48,96) \times$ $0.7 \mathrm{mJy} / \mathrm{beam}$. The resolution is $61^{\prime \prime} \times 51^{\prime \prime}$. 


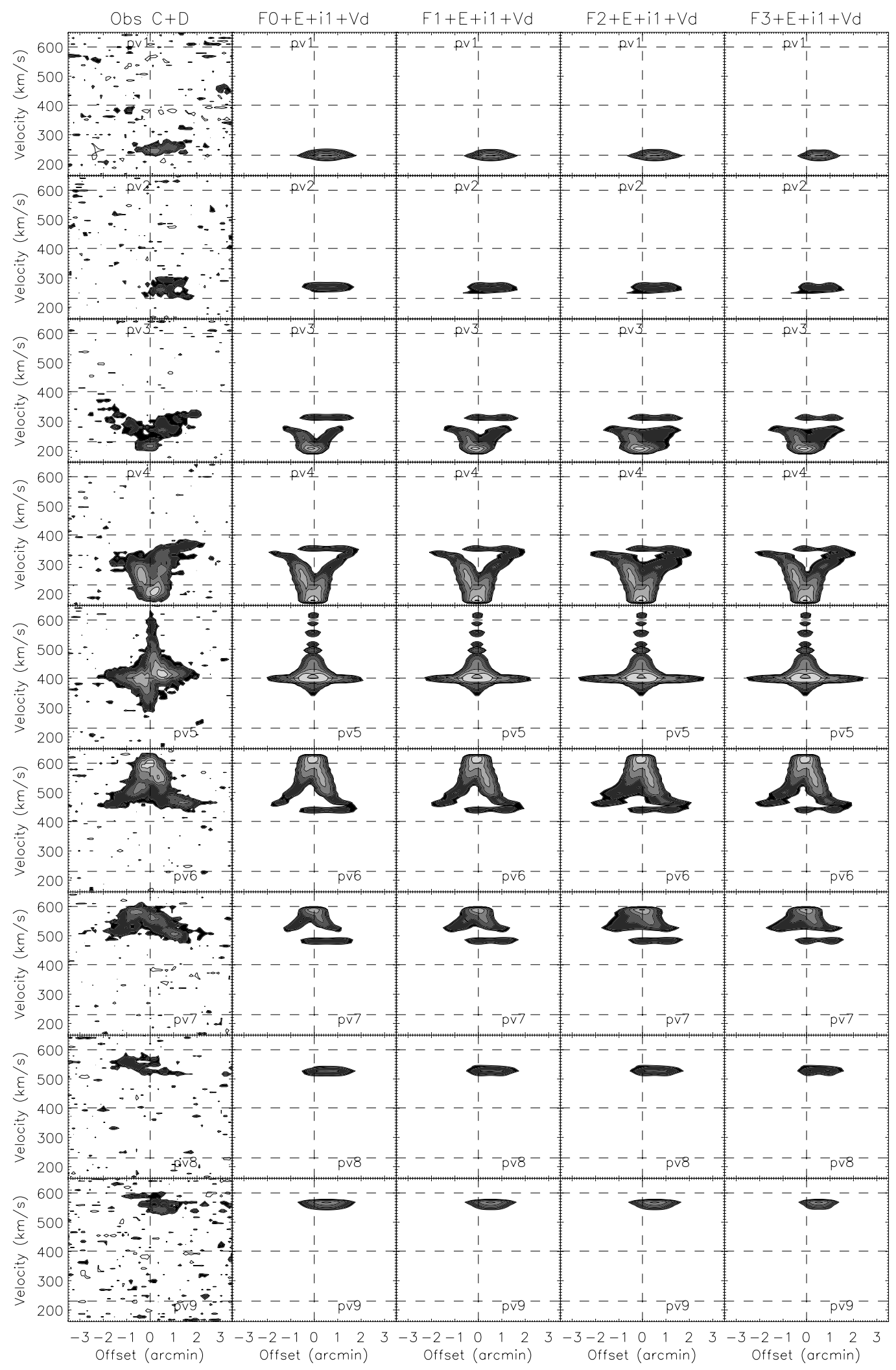

Fig. C.3. NGC $2683 \mathrm{HI}$ C+D array and model position velocity diagrams. The model includes a thin disk, an elliptical component (E), a warp in inclination (i1), a radially decreasing velocity dispersion (Vd), and a flare (F0-F3; see Fig. 9). The contour levels are $(-2,2,3,6,12,24,48,96) \times$ $0.3 \mathrm{mJy} /$ beam. The resolution is $19^{\prime \prime} \times 18^{\prime \prime}$. 


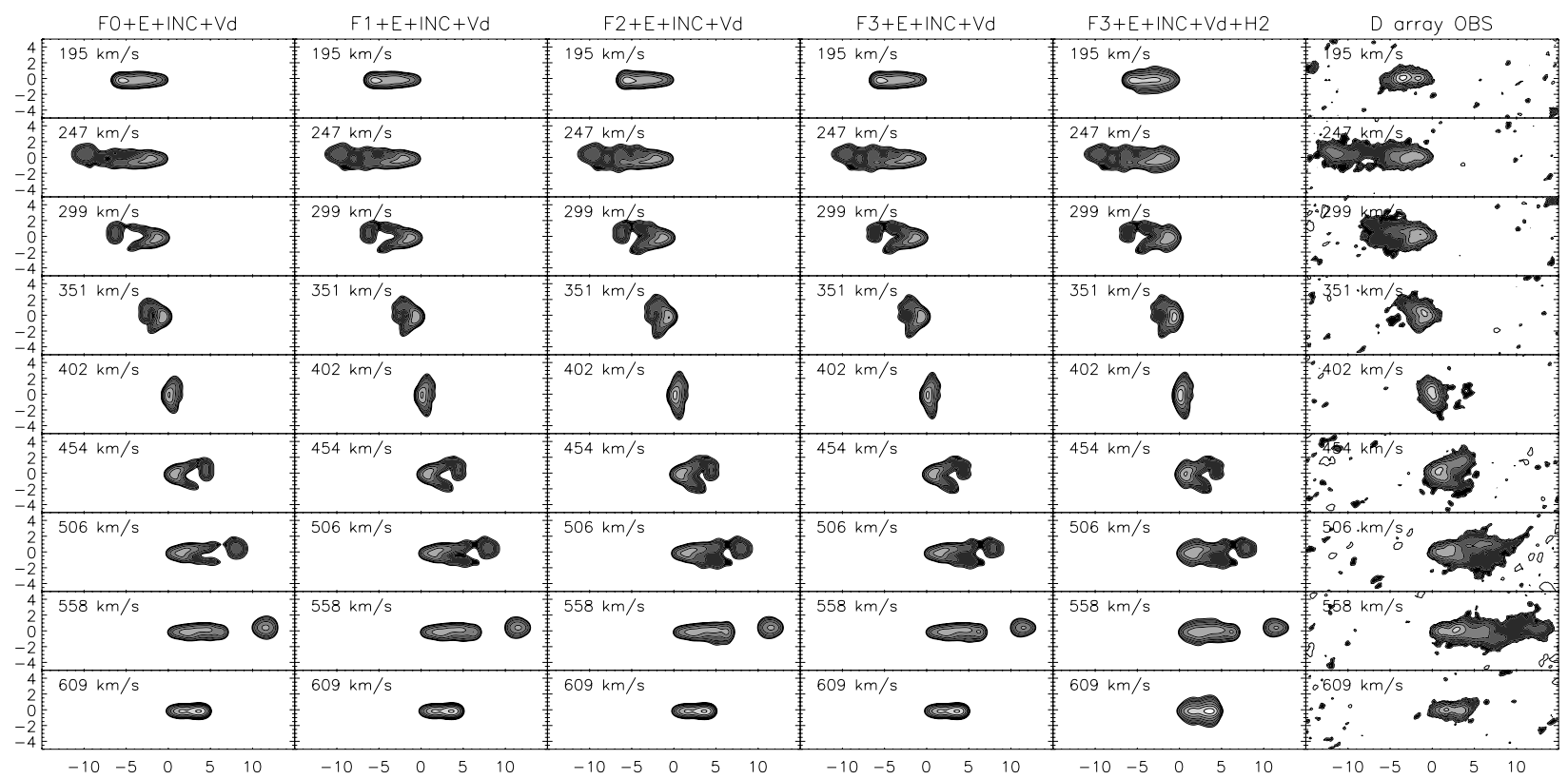

Fig. C.4. Selected HI D array and model channel maps. The model includes a thin disk, an elliptical component (E), a warp in inclination (INC), a radially decreasing velocity dispersion (Vd), a flare (F0-F3; see Fig. 9), and an HI halo (H2). The offsets along the $x$ and $y$ axes are in arcmin. The contour levels are $(-2,2,3,6,12,24,48,96) \times 0.8 \mathrm{mJy} / \mathrm{beam}$. The resolution is $61^{\prime \prime} \times 51^{\prime \prime}$. The galaxy was rotated by $45^{\circ}$.

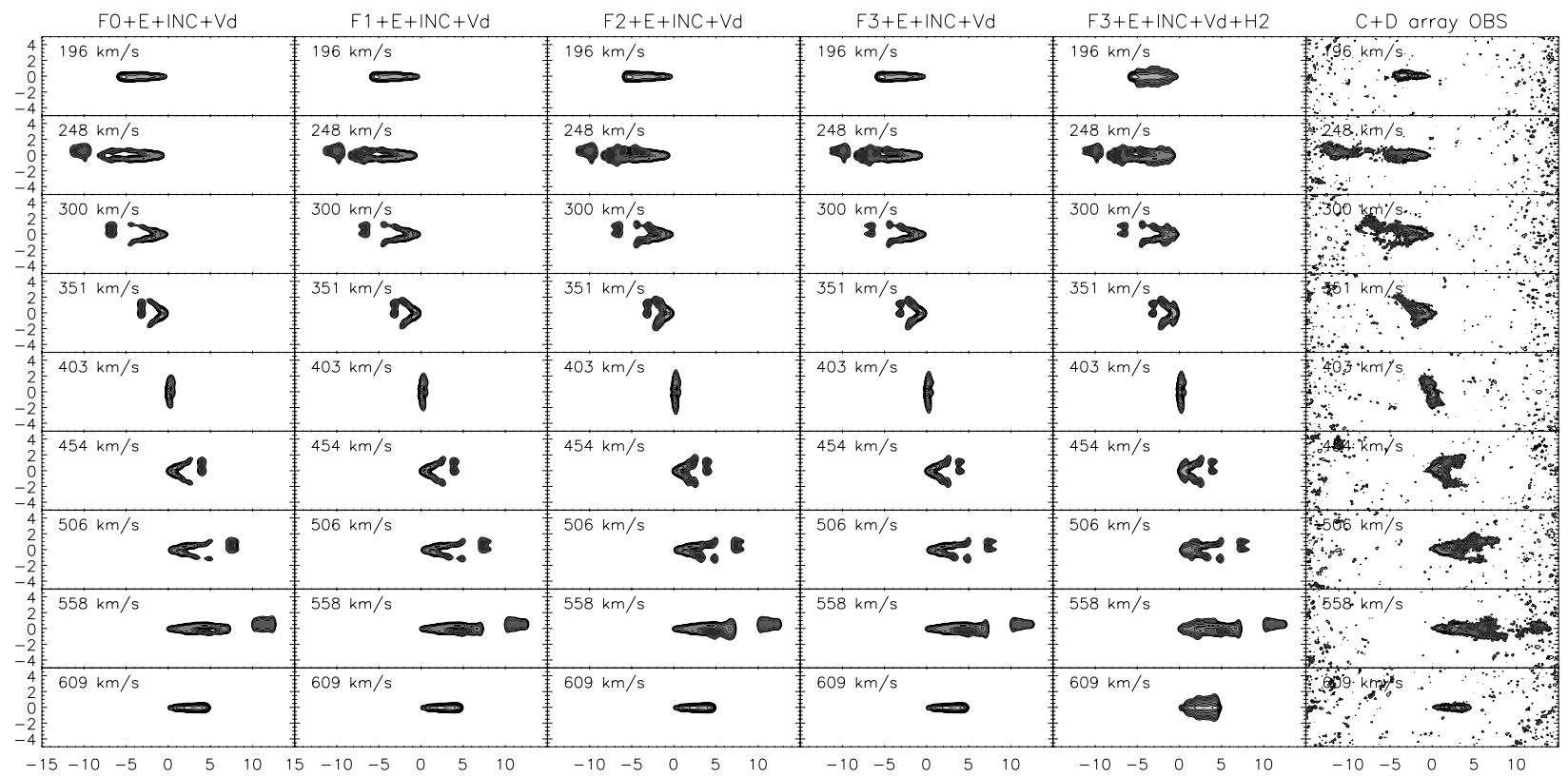

Fig. C.5. Selected HI C+D array and model channel maps. The model includes a thin disk, an elliptical component (E), a warp in inclination (INC), a radially decreasing velocity dispersion (Vd), a flare (F0-F3; see Fig. 9), and an HI halo (H2). The offsets along the $x$ and $y$ axes are in arcmin. The contour levels are $(-2,2,3,6,12,24,48,96) \times 0.4 \mathrm{mJy} / \mathrm{beam}$. The resolution is $19^{\prime \prime} \times 18^{\prime \prime}$. The galaxy was rotated by $45^{\circ}$. 

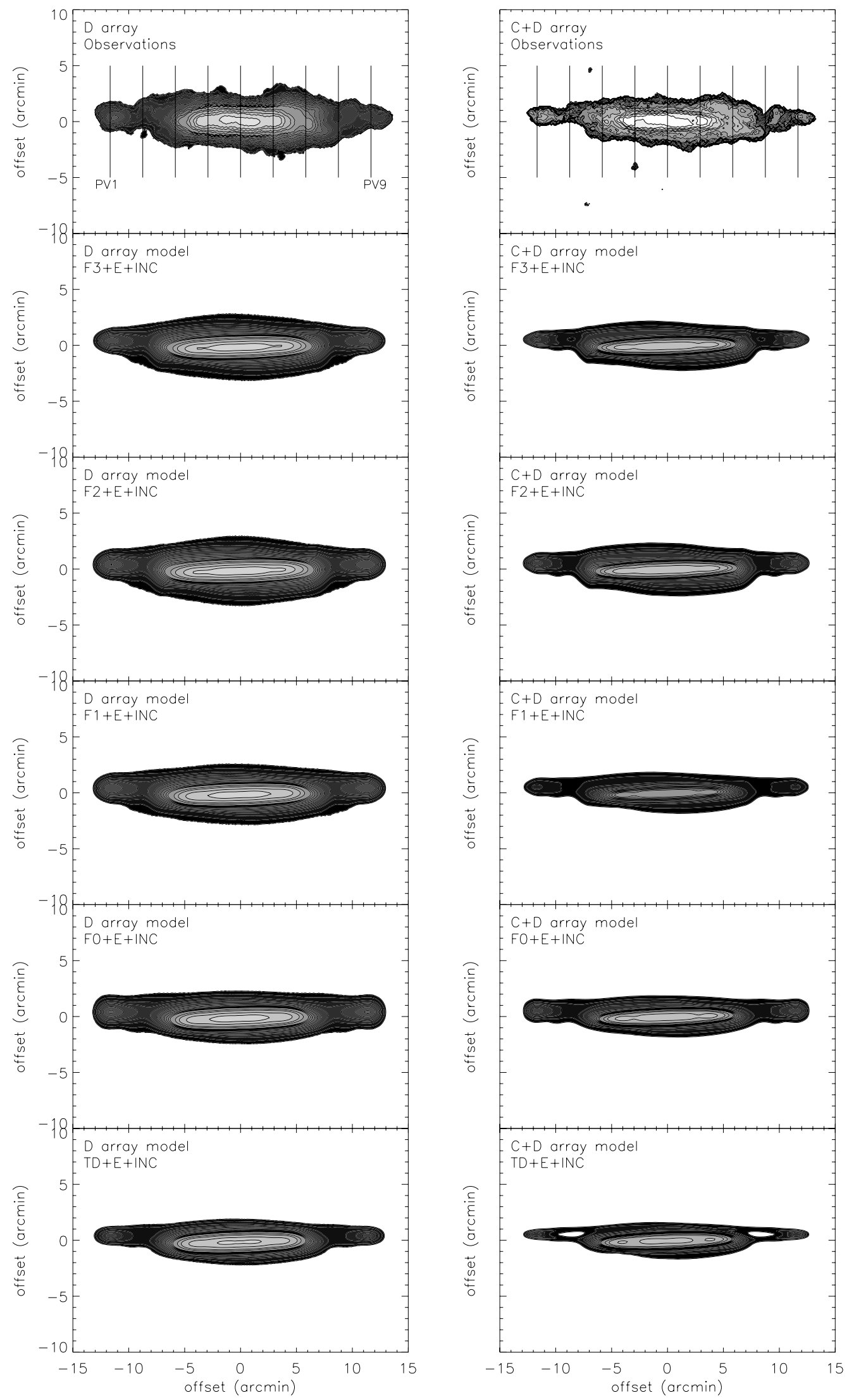

Fig. C.6. Model gas distribution maps. Left column: D array; right column: C+D array data. Upper panels: observations; lower panels: models with an elliptical component (E), a warp in inclination (INC), and the warps F0-F3; best-fit (F3 + warp) model. The contours are the same as for Fig. 2. 


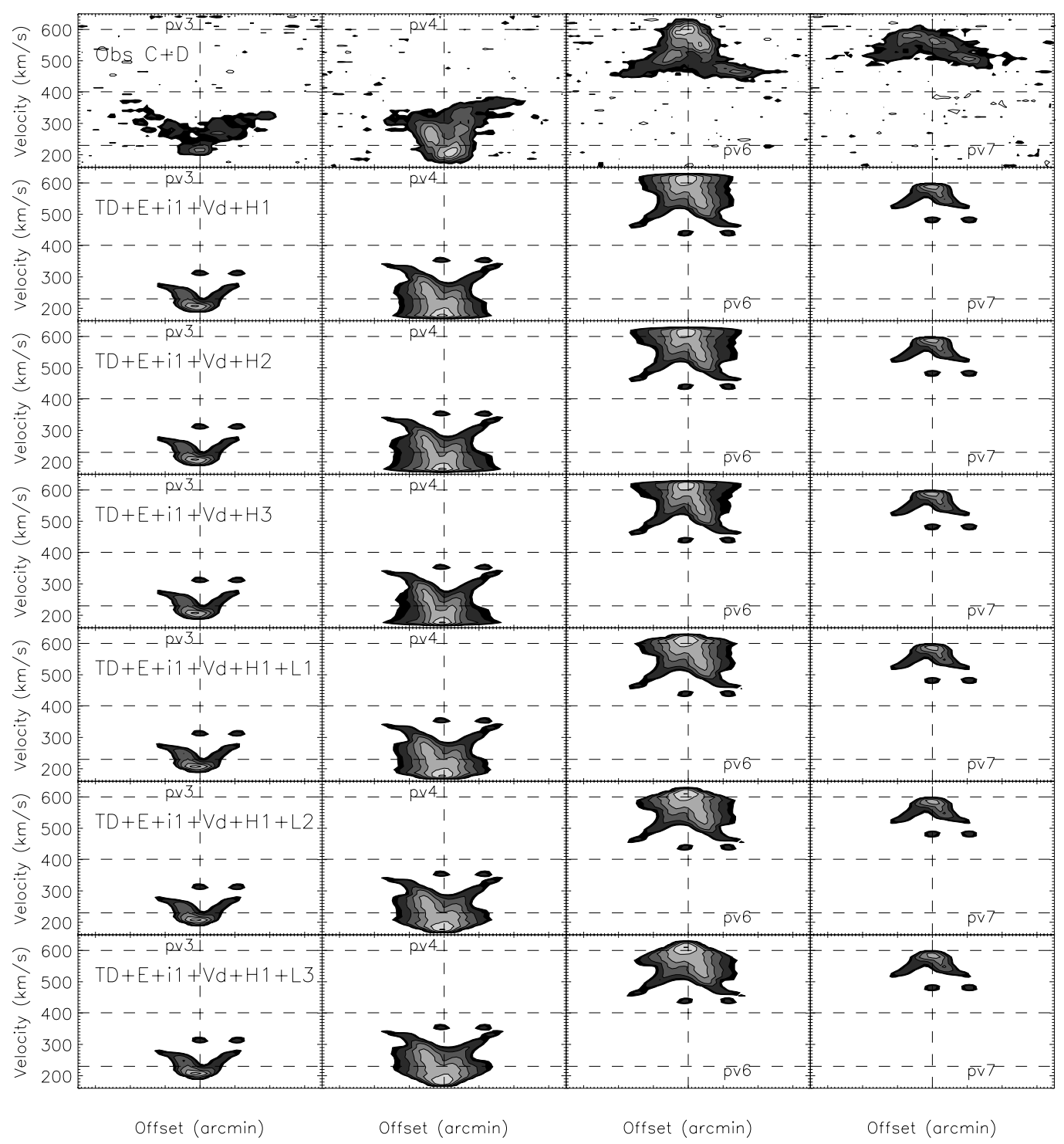

Fig. C.7. Selected position velocity diagrams. C+D observations and models with a thin disk (TD), an elliptical component (E), a warp in inclination (i1), a centrally increasing velocity dispersion (Vd), different HI halos (H1-H3), and different velocity lags (L1, L2, L3: $\left.5,10,15 \mathrm{~km} \mathrm{~s}^{-1} \mathrm{kpc}^{-1}\right)$. The contour levels are $(-2,2,3,6,12,24,48,96) \times 0.3 \mathrm{mJy} / \mathrm{beam}$. The resolution is $19^{\prime \prime} \times 18^{\prime \prime}$. 